\title{
Equipartition of energy, Avogadro law and ratio of specific heats
}

\author{
Mário J. de Oliveira*1] \\ ${ }^{1}$ Universidade de São Paulo, Instituto de Física, São Paulo, SP, Brasil
}

Received on October 31, 2018. Accepted on December 05, 2018.

\begin{abstract}
The equipartition of energy in its simplest form, which is related to the translational motion of the molecules of a gas, was announced independently by Waterston in 1845 and by Clausius in 1857. In its more general form, it was formulated by Maxwell in 1860. Together with the relation between pressure and translational motion, given by the kinetic theory of gases, one can derive the equation of state of an ideal gas. One can also derive the Avogadro law, a fundamental law of physical chemistry as stated by Meyer and Mendeleev. From the equipartition of energy Boltzmann could explain the experimental ratio $\gamma$ of the specific heats of diatomic gases, an explanation that was countered by Maxwell. We discuss these two conflicting explanation, and present an account and a critical analysis of the emergence of the law of equipartition of energy and other laws that preceded it but are understood as consequences or related to it. Our account includes also the Laplace relation between the speed of sound and $\gamma$, and the Clément and Desormes experiment to determine $\gamma$.

Keywords: Equipartition of energy, Avogadro law, ratio of specific heats, diatomic gases, Clément and Desormes experiment
\end{abstract}

\section{Introduction}

Around 1877, Maxwell and Boltzmann were involved with the explanation concerning the specific heat of ideal gases consisting of diatomic molecules [1,2]. From the law of equipartition of energy [3], the ratio $\gamma$ between the specific heat at constant pressure $c_{p}$ and at constant volume $c_{v}$ was known to be $\gamma=(\nu+2) / \nu$, where $\nu$ is the number of degrees of freedom of a molecule. A diatomic molecule has three translational degrees of freedom and, according to Boltzmann, only two rotational degrees of freedom, which gives $\nu=5$, and $\gamma=1.4$. This result was very close to the experimental value of $\gamma$ observed for the diatomic gases such as the atmospheric air. Maxwell countered Boltzmann explanation on the grounds that a diatomic molecule, being an extended body, should have three and not two degrees of rotation. Although Maxwell reasoning was in accordance with Newtonian mechanics, it did not explain the experimental result of $\gamma$. This paradox remained unsolved until the emergence of quantum mechanics.

In spite of the conflicting explanation given by Maxwell and Boltzmann, concerning the diatomic gases, both physicists considered the equipartition of energy to be a significant law that could be derived from the kinetic theory of gases. However, the attempts to derive the law from pure mechanics were unsuccessful. A derivation became possible when probability reasonings were incorporated into kinetic theory. From the probability

*Correspondence email address: oliveira@if.usp.br distributions of equilibrium statistical mechanics, the equipartition of energy follows straightforwardly [3].

The equipartition of energy in its simplest form, which comprises only the translational motion of molecules, was independently formulated by Waterston in 1845 , and by Clausius in 1857, and were stated in their respective papers on the kinetic theory of gases. In a more general form, which involves rotational motion in addition to translational motion, the equipartition law was advanced by Maxwell in 1860 .

Here, we give an account and a critical analysis of the emergence and the development of the equipartition of energy. We also give an account and a critical analysis of other laws that were discovered before the equipartition law, which were found to be directly connected to the equipartition law. These laws are those associated to the ideal gas and comprise the equation of state of an ideal gas, the Avogadro law and other laws related to the specific heats as well as to their ratio. Our account ends around the time Maxwell and Boltzmann gave their respective explanations on the specific heats of molecules. It comprises the period where the caloric theory $4-6$ was the prevailing theory of heat and the first decades after the emergence of thermodynamics 6].

The ideal gas equation of state, which combines the Boyle law and Gay-Lussac law of gas expansion, played a relevant role in the development of the theory of heat and the kinetic theory of gases. No significant deviation of these laws were observed before the extensive and thorough experiments conducted by Regnault starting in the 1840s. The laws related to gases discovered before 
this time should thus be understood as related to ideal gases. These include the law stating that pressure is inversely proportional to its volume established by Boyle, [7], the law of gas expansion and the volume law of chemical combination introduced by Gay-Lussac [8], and the molecular hypothesis postulated by Avogadro [9],

The ratio $\gamma$ between specific heats was the essential feature of Laplace explanation of the velocity of sound in gases 10. A direct experiment to determine $\gamma$ experimentally was provided by Cĺement and Desormes, from which Laplace could test his theory. Once established, the Laplace theory could be used to obtain $\gamma$ from the speed of sound, which was the method adopted by Dulong. It was the value of $\gamma$ determined by Dulong that Mayer used in the method he devised to determine the mechanical equivalent of heat [6].

It is our understanding that the description and explanation of concepts and laws must be given in a way as to preserve the terms originally employed. The replacement of an original term by a modern term runs the risk of modifying or misinterpreting its original meaning. However, when this risk does not exist, we will employ the modern terminology for the sake of brevity and better clarity. This is the case of modern names of substances, and of the terms 'adiabatic' and 'kinetic energy'. We will also employ the notation for partial derivative that uses a subscript to indicate which variable is being taken as constant, and make use of the pressure-volume diagram, even if this resource was not used originally.

It is well known that the measurement of a physical quantity is always made in reference to a certain unit. In the case of heat, usually the unit used was the quantity of heat necessary to raise the temperature of a certain amount of water by one degree. The unit corresponding to raise the temperature of one kilogram of water by one degree centigrade was called a 'calorie', a French word, by Clément before 1825 11. Mayer used the German word 'Calorie' in his paper of 1848 on the mechanical equivalent of heat 11]. In 1852, Faber and Silbermann used the term 'calorie' as a unit of heat based on one gram of water 11. However, it was not common to employ the term 'calorie' and its use became widely spread only during the second half of the nineteenth century. Here, we use the term 'calorie', abbreviated 'cal', in the sense given by Faber and Silbermann, even to refer to results given in papers where the word is not explicit mentioned. We also use the abbreviated terms for other units such as ${ }^{\circ} \mathrm{C}$ for degree centigrade, and $\ell$ for liter.

\section{Ideal gas}

\subsection{Equation of state}

The ideal gas played a major role in the development of the theory of heat and kinetic theory of gases. Its equation of state was a result of investigations on the elastic and thermal properties of gases that started in the middle of the seventeenth century, after the discovery of the atmospheric pressure and the invention of the barometer. Experiments conducted by Boyle on elastic properties of air, reported in 1662, led him to the law that the pressure of a certain amount of air is inversely proportional to its volume 12, as can be seen in Figure 1] where we have plotted Boyle's original data in the pressure-volume diagram. Mariotte has also reached the same law but the publication of his result occurred seventeen years after that of Boyle 13.

Experiments on the expansion of gases by heat carried out by Gay-Lussac allowed him to announce in 1802 the following law: under the same increment of temperature, all gases expand by the same amount, provided they are subjected to the same conditions 14. Dalton also arrived at a similar law but Gay-Lussac's experiments were performed more carefully, taking the precaution of working with dry gases. The choice of gases and the temperature range studied by Gay-Lussac was better, and the results were more clearly stated 8 . Sometimes the law is unduly attributed to Charles because GayLussac mentioned by chance some unpublished results of Charles 8.

The law of expansion announced by Gay-Lussac refers to the fraction of volume and not to the volume itself. If the volume increases from $v$ to $v^{\prime}$ when the temperature increases from $\theta$ to $\theta^{\prime}$, at constant pressure, the fraction of volume increased per unit of temperature is

$$
\alpha=\frac{1}{v} \frac{v^{\prime}-v}{\theta^{\prime}-\theta}
$$

Gay-Lussac measured the fraction increase $\left(v^{\prime}-v\right) / v$ between $\theta=0{ }^{\circ} \mathrm{C}$ and $\theta^{\prime}=100{ }^{\circ} \mathrm{C}$ for several gases including atmospheric air, hydrogen, oxygen, nitrogen,

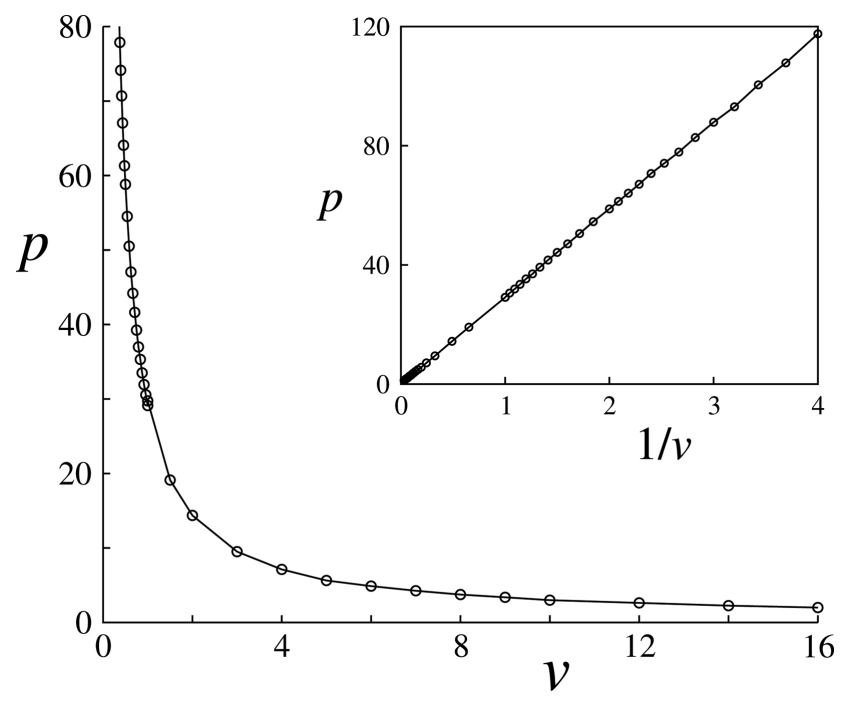

Figure 1: Pressure $p$, in inches of mercury, versus volume $v$ of atmospheric air from experimental data published by Boyle in 1662 [12]. The unit of volume is that in which $v=1$ at the atmospheric pressure. The inset shows the same data plotted in the diagram $p$ versus $1 / v$. 
carbon dioxide, and ammonia, and found the same value $\alpha=0.00375$ per ${ }^{\circ} \mathrm{C}$ for these gases $[14$. The apparatus used by Gay-Lussac is shown in Figure 2.

According to an account given by Biot in 1816 [15], Gay-Lussac also established that the expansion of a gas at a constant pressure is proportional to the expansion of mercury, which means to say that the volume varies linearly with temperature in an isobaric expansion. From this result, it follows that $\alpha$, given by (1) does not depend on the difference $\theta^{\prime}-\theta$ but depends only on the initial temperature $\theta$, so that $\alpha$ is also given by $(1 / v)(\partial v / \partial \theta)_{p}$.

Poisson 16 combined the Boyle and Gay-lussac laws into a single equation in the form

$$
p=b \rho\left(1+\alpha_{0} \theta\right)
$$

where $p$ is the pressure, $\rho$ is the density, that is, the mass per unit volume, $\theta$ is the temperature in degrees centigrade, and $b$ and $\alpha_{0}$ are constants. Carnot 17, in his memoir on the production of work by heat, also combined the Boyle and Gay-lussac laws into a single equation and was followed in this matter by Clapeyron [18] who expressed the combined law in the form 1

$$
p v=r\left(\theta_{0}+\theta\right),
$$

where $v=1 / \rho$ is the volume per unit mass, $\theta$ is the temperature also in degrees centigrade, and $r$ and $\theta_{0}$ are

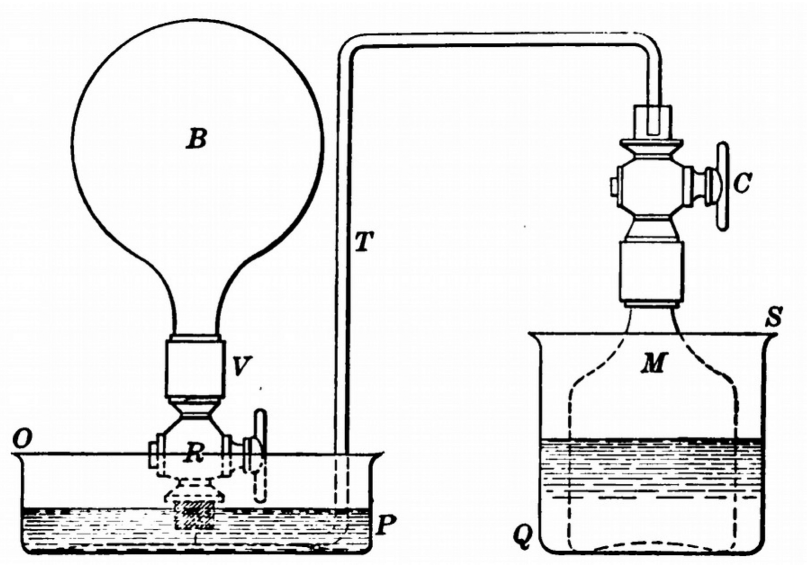

Figure 2: Apparatus used by Gay-Lussac in the experiment on thermal expansion of gases reported in 1802 [14]. Gas coming from the bell jar $M$ is introduced into the flask $B$. With the tap $\mathrm{R}$ opened, the flask is heated and part of the gas escapes from the flask. When the temperature reaches $100{ }^{\circ} \mathrm{C}$, the tap $\mathrm{R}$ is closed and then the flask is submerged in water with ice. When it reaches the temperature of $0{ }^{\circ} \mathrm{C}$, the tap $\mathrm{R}$ is opened and a certain amount of water flushes inside the flask. The volume of water that entered the flask equals the increase in volume of the gas when it is heated from 0 to $100{ }^{\circ} \mathrm{C}$. The volume of water is measured by weighting the flask $B$ with water. Figure from reference [14].

${ }^{1}$ Clapeyron wrote $p v=R(267+t)$. The constant $R$ in this expression should not be confused with the universal gas constant. To avoid confusion we are using in its place the letter $r$. The employing of letter $R$ by Clapeyron may have induced the use of the same letter $R$ to represent the universal gas constant 19 . constants. Comparing equations (2) and (3), we see that the constants $\alpha_{0}$ and $\theta_{0}$ are related by $\theta_{0}=1 / \alpha_{0}$.

From equation (3), it follows that the coefficient of thermal expansion $\alpha_{p}=(1 / v)(\partial v / \partial \theta)_{p}$ is

$$
\alpha_{p}=\frac{1}{\theta_{0}+\theta}=\frac{\alpha_{0}}{1+\alpha_{0} \theta}
$$

At $0{ }^{\circ} \mathrm{C}$, the coefficient of thermal expansion is $\alpha_{0}=1 / \theta_{0}$ and, according to Gay-Lussac, independent of the nature of the gas. The value found by Gay-Lussac, $\alpha_{0}=0.00375$ per ${ }^{\circ} \mathrm{C}$, gives $\theta_{0}=267^{\circ} \mathrm{C}$, after rounding, which was the value used by Carnot and Clapeyron. In an experiment carried out by Rudberg [20], reported in 1837, he found $\alpha_{0}=0.003646$ per ${ }^{\circ} \mathrm{C}$ for the air, which gives $\theta_{0}=274.3$ ${ }^{\circ} \mathrm{C}$.

In his first paper on heat 21], Clausius also used the form (3) with the rounded value $\theta_{0}=273{ }^{\circ} \mathrm{C}$, taken from Regnault experimental results 22 . The value found by Regnault for the thermal expansion for the air was $\alpha_{0}=$ 0.003665 per ${ }^{\circ} \mathrm{C}$, which gives $\theta_{0}=272.9{ }^{\circ} \mathrm{C}$. Regnault also confirmed that $\theta_{0}$ is independent of the nature of the gas by determining $\alpha_{0}$ for several gases [22], some of them presented in Table 1. It becomes thus legitimate to define an absolute scale of temperature $T=\theta_{0}+\theta$, which does not depend on the gas being measured. The equation of state of an ideal gas can then be written as

$$
p v=r T
$$

where only the constant $r$ depends on the nature of the gas. In this form, the equation of state was used by Clausius in 1862 23. It can also be written in the equivalent form used by Laplace [24],

$$
p=r \rho T \text {. }
$$

To determine the constant $r$ it suffices to measure the density $\rho$ for a given temperature and pressure. At 0 ${ }^{\circ} \mathrm{C}$ and the pressure of $1 \mathrm{~atm}$, Biot and Arago reported in 1806 the experimental value $\rho=1 / 773=1.292307$ $\mathrm{g} / \ell$ for the atmospheric air 25]. In 1809, Gay-Lussac

Table 1: Column 2: Thermal expansion $\alpha_{0}$ per ${ }^{\circ} \mathrm{C}$ according to Regnault 22]. Column 3: Temperature $\theta_{0}=1 / \alpha_{0}$ in ${ }^{\circ} \mathrm{C}$, obtained from column 2. Column 4 and 5: Density $\rho$ in $\mathrm{g} / \ell$ at $0^{\circ} \mathrm{C}$ and $1 \mathrm{~atm}$ according to Gay-Lussac [26] and Biot and Arago [25]; and according to Regnault [27,28].

\begin{tabular}{lcccc}
\hline gas & $100 \alpha_{0}$ & $\theta_{0}$ & $\rho$ & $\rho$ \\
\hline air & 0.3665 & 272.85 & 1.29230 & 1.29318 \\
oxygen & & & 1.42617 & 1.42980 \\
nitrogen & 0.36682 & 272.61 & 1.25241 & 1.25616 \\
hydrogen & 0.36678 & 272.64 & 0.09461 & 0.08957 \\
carbon monoxide & 0.36667 & 272.72 & 1.25072 & 1.2510 \\
nitric oxide & & & 1.33929 & 1.3436 \\
hydrogen chloride & 0.36812 & 271.65 & 1.651 & 1.6131 \\
carbon dioxide & 0.36896 & 271.03 & 1.9637 & 1.97741 \\
nitrous oxide & 0.36763 & 272.01 & 1.96549 & 1.9721 \\
sulfur dioxide & 0.36696 & 272.51 & 2.927 & 2.9057 \\
year & 1842 & 1842 & 1809 & $1847 / 53$ \\
\hline
\end{tabular}


published a list of densities of several gases obtained experimentally by other researchers [26]. These densities, which were given in units where the density of air is unity, multiplied by the density of air given by Biot and Arago yield the densities in $\mathrm{g} / \ell$ shown in Table 1 . For the same conditions, Regnault reported in 1847 the value $\rho=1.293187 \mathrm{~g} / \ell$ for the atmospheric air 27 . The values of $\rho$ obtained by Regnault for other gases [27, 28] are presented in Table 1 .

Deviations from the Boyle and Gay-Lussac laws were observed only after 1840 when Regnault started to carry out extensive and careful experiments on the expansion and thermal properties of gases 22 . In the conclusion of his report on the expansion of gases of 1842, Regnault pointed out that in spite of the deviations, these laws should not be excluded from science since they remain valid in the limit of great dilatation, when the deviations become negligible [22]. This is the case of permanent gases, which are those that do not condense by the action of pressure at room temperatures, such as atmospheric air, oxygen, nitrogen, and hydrogen. The state of a gas at great dilatation, when the Boyle and Gay-Lussac becomes valid, Regnault called a perfect gaseous state 22 . Rankine referred to a gas obeying these two laws as a 'perfect gas' [29] and Clausius as a 'perfect gas' or as an 'ideal gas' 30 .

\subsection{Specific heat}

The distinction between the concepts of quantity of heat and intensity of heat, or temperature, which arose in the middle of the eighteenth century [4, gave origin to the concept of heat capacity, which is the quantity of heat necessary to increase the temperature of a body by one unit. The first attempt to measure the heat capacity of gases was made by Crawford in 1777, but despite of his considerable experimental ability the results obtained were insignificant 5]. Crawford measurements were made at constant volume and he believed that the heat capacity would be distinct if the volume of the gas was allowed to change. A clear distinction between heat capacity at constant pressure and at constant volume was provided by Haüi in 1806, who also argued that the former should be greater than the latter [31].

Usually, the heat capacity measured was that at constant pressure. The experimental determination of the heat capacity of gases at constant volume offered a difficulty that was not overcome before the 1880s [5]. Only indirect methods could give reliable values of the heat capacity at constant volume, such as the experimental determination of the ratio between the two kinds of heat capacities.

The determination of the specific heat, the heat capacity per unit mass, of gases was the subject of the prize of the French Academy of Sciences for the year 1812, motivated by its relevance in characterizing the thermal properties of gases and by the discrepancies of previous measurements 5. The prize was won by Delaroche and Bérard for their measurements of the specific heat of several gases including the atmospheric air [32]. They measured the specific heat at constant pressure $c_{p}$, finding for the air the value $c_{p}=0.2669 \mathrm{cal} / \mathrm{g}^{\circ} \mathrm{C}$. For other gases, they first measured the specific heats relative to that of the atmospheric air, and then multiplied these values by the specific heat of air. Their results are shown in Table 2

To find the specific heat of a substance one needs to measure both the mass and the heat capacity itself. If instead of mass, one measures volume, which is easier in the case of gases, one could determine the volume specific heat, which is the heat capacity per unit volume. The volume specific heat at constant pressure $\bar{c}_{p}$ is related to the ordinary specific heat by $\bar{c}_{p}=c_{p} \rho$, where $\rho$ is the gas density. In their prize paper, Delaroche and Bérard used this relation to calculate the volume specific heat of some gases, shown in Table 2 They also discussed the opinion of some physicists that the volume specific heat might be the same for all gases, but they gave a negative answer to this matter in view of their results.

The volume specific heat of some gases was also the object of measurement by Haycraft and by Delarive and Marcet. The experiments conducted by Haycraft in 1823 [33] led him to state that the volume specific heat of

Table 2: Specific heat $c_{p}$ and volume specific heat $\bar{c}_{p}$ of gases obtained experimentally by Delaroche and Bérard (column 2 and 4) [32], by Delarive and Mercet [37] (column 5), and by Regnault (columns 3 and 6) [38]. Specific heat $c_{p}$ in cal $/ \mathrm{g}^{\circ} \mathrm{C}$. Volume specific heat $\bar{c}_{p}$ in units in which that of air is unity.

\begin{tabular}{lccccc}
\hline gas & $c_{p}$ & $c_{p}$ & $\bar{c}_{p}$ & $\bar{c}_{p}$ & $\bar{c}_{p}$ \\
\hline air & 0.2669 & 0.2375 & 1 & 1 & 1 \\
oxygen & 0.2361 & 0.2175 & 0.9765 & 1 & 1.0126 \\
nitrogen & 0.2754 & 0.2438 & 1 & 1 & 0.9971 \\
hydrogen & 3.2936 & 3.4090 & 0.9033 & 1 & 0.9933 \\
carbon monoxide & 0.2884 & 0.2450 & 1.0340 & & 0.9979 \\
nitric oxide & & 0.2317 & & & 1.0131 \\
hydrogen chloride & & 0.1852 & & & 0.9823 \\
carbon dioxide & 0.2210 & 0.2163 & 1.2583 & 1.222 & 1.3924 \\
nitrous oxide & 0.2369 & 0.2262 & 1.3503 & & 1.4514 \\
sulfur dioxide & & 0.1544 & & & 1.4375 \\
ethylene & 0.4207 & 0.4040 & 1.5530 & 1.531 & 1.7288 \\
year & 1813 & 1853 & 1813 & 1835 & 1853 \\
\hline
\end{tabular}


atmospheric air, hydrogen, oxygen, nitrogen, and carbon dioxide were the same. Delarive and Marcet, based on their experimental results, reported in their publications of 1827 and 1829 [34,35, extended Haycraft results to all gases, in opposition to Delaroche and Bérard conclusion. Later on, in 1835, taking into account the critics of Dulong [36], and conducting new measurements, whose results are shown in Table 2 . Delarive and Marcet restricted this law to two classes of gases, stating that [37]:

(1) At the same temperature and under the same pressure, the simple gases and compound gases which result from the combination of simple gases without a change of volume have the same volume specific heat.

The gases that are object of this law are identified as diatomic gases.

Precise measurements of $c_{p}$ were carried out by Regnault in 1853 28, 38. He measured the specific heat of several gases finding for air the value $c_{p}=0.2379$ $\mathrm{cal} / \mathrm{g}^{\circ} \mathrm{C}$. Values of the specific heat of some other gases determined experimentally are shown in Table 2. These experimental results of Regnault showed that the differences on the volume specific heats of simple gases as well as of some compound gases are very small, as seen in Table 2 leading him to accept law (1) of Delarive and Marcet, as long as the gases obey the ideal gas equation. Regnault commented that this law is an extension of the Dulong and Petit law 39 according to which the product $c_{p} \mu$, where $\mu$ denotes the atomic weight, is the same for simple solid bodies. Indeed, the volume specific heat is $\bar{c}_{p}=c_{p} \rho$ and the gas density $\rho$ is proportional to $M$ so that $\bar{c}_{p}$ is proportional to $c_{p} \mu$. Since law (1) of Delarive and Marcet states that $\bar{c}_{p}$ is the same, one concludes that the product $c_{p} \mu$ is also the same. The proportionality between $\rho$ and $\mu$ was taken for granted by Regnault, but as we shall see it is a consequence of Avogadro law.

Based on his experimental data on the specific heat of gases obtained in 1853 [38], Regnault could infer the following laws concerning the ideal gases:

(2) The specific heat does not depend on the volume.

According to Regnault, this result shows that the heat capacity of an ideal gas is independent of the distances that separate the gas particles. This law is equivalent to say that the specific heat of an ideal gas depends only on the temperature.

Regnault could also infer the following law [38]:

(3) The specific heats of the atmospheric air and hydrogen do not vary with temperature.

This result was based on measurements on air in the interval between -30 and $200{ }^{\circ} \mathrm{C}$, and on hydrogen between 0 and $200{ }^{\circ} \mathrm{C}$.

\subsection{Expansion and contraction}

When air is rapidly compressed inside a vessel, its temperature increases. If the vessel is in thermal contact with the environment, then after the compression, heat is released to outside and the gas reaches the environment temperature. The representation of this process is shown in Figure 3. Analogously, if the gas is expanded, its temperatures decreases, and after that it reaches the temperature by absorbing heat from the outside. During the rapidly contraction or dilatation, no heat is involved. This phenomenon of heating by a rapid compression and cooling by a rapid dilatation was described by Cullen in 1755 and independently by Darwin in 1788 [5].

In 1829 , in his paper on the ratio of the specific heats of gases, Dulong advanced a law concerning the expansion and contraction of gases [36]:

(4) Equal volumes of all gases taken at the same temperature and under the same pressure, being compressed or suddenly dilated by the same fraction of their volume, give off or absorb the same absolute quantity of heat.

A similar law was advanced by Carnot in his book on the theory of heat published in 1824 [17], but the gas undergoes an isothermal process:

(5) When a gas expands isothermally, from a given volume and a given pressure until a final volume, the quantity of heat absorbed is independent of the nature of the gas.

This law is a consequence of the Carnot theory of heat applied to a gas obeying Boyle and Gay-Lussac laws, as we shall see below.

The theory of Carnot was developed in an analytical form by Clapeyron in his memoir on heat of 1834 [18]. In this memoir, he states that law (5) was demonstrated experimentally by Dulong. Since Dulong advanced law

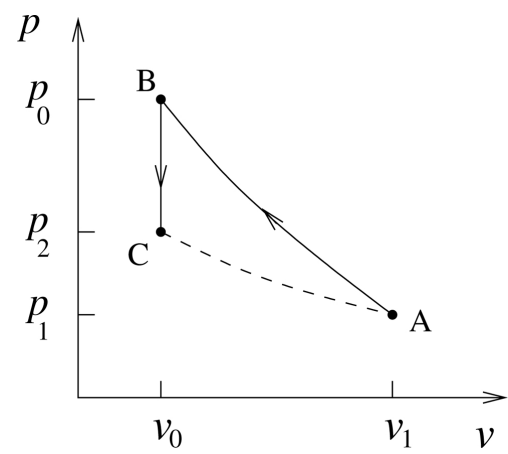

Figure 3: Representation in the $p-v$ diagram of a sudden compression of a gas inside a vessel with heat conducting walls. The gas is compressed adiabatically from state $A$ to $B$, causing an increase in its temperature. From $B$ to $C$, the gas delivers heat at constant volume and its temperature decreases. At states $A$ and $C$ the gas has the same temperature, that is, the dashed line is an isotherm. 
(4), and not law (5), it is implied that Clapeyron was considering law (5) as equivalent to law (4). To understand Clapeyron, we should bear in mind that his reasoning was based on caloric theory, that considered heat as a state function. Accordingly, the heat delivered by the gas should not depend on the process the gas is undergoing but only on the final and the initial states. The conclusion is that, within the caloric theory, the heat delivered along the process $\mathrm{ABC}$ is the same as that given off by the isotherm AC shown in Figure 3 The former process is related to law (5) and the latter to law (4).

Law (5) was derived in an analytical form by Clapeyron from Carnot theory, which assumes that the heat per unit mass $q$ is a state function, which means to say that $q(v, p)$ is a function of two independent variables chosen by Clapeyron to be $v$ and $p$. Together with the equation of state $p v=r T$, where $T=\theta_{0}+\theta$, Clapeyron could derive the following equation that gives the amount of heat $q$ per unit mass in a gas 18

$$
q=r C \ln v+A
$$

where $C$ is a universal function of temperature $\theta$ introduced by Carnot, and $A$ depends on the temperature. A derivation of this equation is also given in reference [6]. From equation (7), we see that the isothermal increase in heat $q^{*}$ between states with volumes $v$ and $v^{\prime}$ is

$$
q^{*}=r C \ln \left(v^{\prime} / v\right)
$$

If at the initial state, $p, v$ and $T$ are the same for all gases, it follows from the ideal gas equation, $p v=r T$, that $r$ has the same values for all gases, and equation (8) becomes the mathematical expression of law (5). This equation is also the mathematical expression of law (4) because $q^{*}$ in equation (8) does not depend on the process but only in the initial and final state.

Another consequence of the theory of Carnot applied to a gas obeying the Boyle and Gay-Lussac laws concerns the difference between the two specific heats. This consequence was stated by Carnot as follows [17]:

(6) The difference between the volume specific heat at constant pressure and constant volume is the same for all gases at the same pressure and temperature.

Within Carnot theory, the specific heats are given by $c_{v}=(\partial q / \partial \theta)_{v}$ and $c_{p}=(\partial q / \partial \theta)_{p}$. From these definitions, Clapeyron could find from equation (7) that the difference between the volume specific heats $\bar{c}_{p}=\rho c_{p}$ and $\bar{c}_{v}=\rho c_{v}$ is

$$
\bar{c}_{p}-\bar{c}_{v}=\frac{p C}{T^{2}}
$$

which is the mathematical expression of law (6) because $C$ does not depend on the nature of the gas.

\section{Ratio of specific heats}

\subsection{Laplace}

In the first years of the nineteenth century, Laplace conceived the idea that the rarefaction and compression occurring in the propagation of sound in the air are processes taking place without the exchange of heat [10]. This idea was distinct from that implicit in the calculation of the speed of sound by Newton in which the rarefaction and compressions are isothermal. Based on this idea, Laplace announced in a publication of 1816 40 that speed of sound $v_{\mathrm{s}}$ in a gas is the product of the speed of sound given by Newton formula $\sqrt{p / \rho}$ and the square root of the ratio

$$
\gamma=\frac{c_{p}}{c_{v}}
$$

between the specific heats, that is,

$$
v_{\mathrm{s}}=\sqrt{\gamma p / \rho}
$$

where $p$ is the pressure of the gas and $\rho$ its density. The factor $\gamma$ in this formula was viewed as a correction to the formula advanced by Newton. A derivation of formula (11) within the caloric theory was given by Laplace in 1822 [24].

The speed of sound can be determined theoretically from the wave equation that governs the changes of pressure and densities of an elastic medium. In his treatise on analytical mechanics, published in 1788 [41], Lagrange derived from Newtonian mechanics the wave equation for the propagation of sound and, assuming that the ratio $p / \rho$ between pressure and density is constant, he obtained Newton formula for the speed of sound. From the ideal gas equation, $p / \rho$ is proportional to the temperature implying that, in Lagrange derivation, the variations in density and pressure occurs at constant temperature.

Stimulated by Laplace, Biot $[42$ examined in 1802 the influence that the variations of temperature that accompany the dilatations and compressions of air might have on the speed of sound. Assuming that $p$ is a generic function of $\rho$, and following a derivation similar to that of Lagrange, Biot found that the speed of sound $v_{\mathrm{s}}$ in a gas is the square root of $\partial p / \partial \rho$. If the variation is isothermal then $v_{\mathrm{s}}^{2}$ equals $(\partial p / \partial \rho)_{\theta}$, which for an ideal gas is $p / \rho$ and we get Newton formula. According to Laplace, the variation occurs without the intervention of heat so that

$$
v_{\mathrm{s}}^{2}=\left(\frac{\partial p}{\partial \rho}\right)_{q},
$$

where the index $q$ indicates that the derivative is carried out at constant heat.

In the following we give a derivation of formula (11) using a procedure equivalent to that of Laplace [24], starting from equation (12) and taking into account that, within the caloric theory, heat is a state function. Following Laplace the heat per unit mass $q(\rho, p)$ is as a function of the independent variables $\rho$ and $p$. Using the 
usual rules of differential calculus, one finds

$$
\begin{aligned}
& \left(\frac{\partial p}{\partial \rho}\right)_{q}=-\frac{(\partial q / \partial \rho)_{p}}{(\partial q / \partial p)_{\rho}} \\
& \left(\frac{\partial p}{\partial \rho}\right)_{\theta}=-\frac{(\partial \theta / \partial \rho)_{p}}{(\partial \theta / \partial p)_{\rho}}
\end{aligned}
$$

Taking the ration of these two equations we get

$$
\frac{(\partial p / \partial \rho)_{q}}{(\partial p / \partial \rho)_{\theta}}=\frac{(\partial q / \partial \theta)_{p}}{(\partial q / \partial \theta)_{\rho}}
$$

But the right-hand side of this equation is the ratio $c_{p} / c_{v}=\gamma$ from which we find

$$
\left(\frac{\partial p}{\partial \rho}\right)_{q}=\gamma\left(\frac{\partial p}{\partial \rho}\right)_{\theta} .
$$

From the equation of an ideal gas, we find $(\partial p / \partial \rho)_{\theta}=$ $p / \rho$, which replaced into equation 16 gives

$$
\left(\frac{\partial p}{\partial \rho}\right)_{q}=\gamma \frac{p}{\rho}
$$

which allows us to reach Laplace formula for the velocity of sound in a gas.

It should be remarked that the ratio $\gamma$ of the specific heats is identical to the ratio $\kappa_{\theta} / \kappa_{q}$ between the isothermal and the adiabatic compressibilities, that is,

$$
\frac{\kappa_{\theta}}{\kappa_{q}}=\frac{c_{p}}{c_{v}}
$$

The identity follows directly from equation $(16)$ and from the definitions $\kappa_{\theta}=(1 / \rho)(\partial \rho / \partial p)_{\theta}$ and $\kappa_{q}=(1 / \rho)(\partial \rho / \partial p)_{q}$ From equation 12 we see that the speed of sound is related to the adiabatic compressibility, $v_{\mathrm{s}}^{2}=1 / \rho \kappa_{p}$.

\subsection{Poisson}

The variations of temperature involved in the oscillations in density and pressure in the propagation of sound was also examined by Poisson in 1807 [43]. He used the same method employed by Biot to show that the speed of sound is the square root of $\partial p / \partial \rho$. Following Laplace, he assumes that the variations in pressure and densities are adiabatic, which implies the result 12 for the speed of sound. From 12 , he derives the formula

$$
v_{\mathrm{s}}=\sqrt{(p / \rho)(1+k)}
$$

where $k$ was a correction factor of Newton formula, given by

$$
k=\frac{1 / \alpha_{q}}{\theta_{0}+\theta},
$$

where $\alpha_{q}=-(1 / v)(\partial v / \partial \theta)_{q}$ is the fraction of volume increased adiabatically per unit of temperature.

In the following we derive results $(19)$ and 20 using a procedure equivalent to that of Poisson, which assumes, in accordance to the caloric theory, that $q$ is a state function, that is, $q(\rho, p)$ is a function of the density $\rho$ and the pressure $p$. Using the usual rules of differential calculus,

$$
\begin{gathered}
\left(\frac{\partial p}{\partial \rho}\right)_{q}=\left(\frac{\partial p}{\partial \rho}\right)_{\theta}+\frac{(\partial p / \partial \theta)_{\rho}}{(\partial \rho / \partial \theta)_{q}} \\
\left(\frac{\partial p}{\partial \theta}\right)_{\rho}=-\left(\frac{\partial \rho}{\partial \theta}\right)_{p}\left(\frac{\partial p}{\partial \rho}\right)_{\theta},
\end{gathered}
$$

which replaced in 21 , gives

$$
\left(\frac{\partial p}{\partial \rho}\right)_{q}=(1+k)\left(\frac{\partial p}{\partial \rho}\right)_{\theta},
$$

where

$$
k=-\frac{(\partial \rho / \partial \theta)_{p}}{(\partial \rho / \partial \theta)_{q}} .
$$

Recalling that $\rho=1 / v$ we may write

$$
k=\frac{\alpha_{p}}{\alpha_{q}}
$$

where $\alpha_{p}=(1 / v)(\partial v / \partial \theta)_{p}$ is the fraction of volume increased per unit of temperature at constant pressure.

For an ideal gas $\alpha_{p}=1 /\left(\theta_{0}+\theta\right)$, which gives formula 20 , and $(\partial p / \partial \rho)_{\theta}=p / \rho$, which gives

$$
\left(\frac{\partial p}{\partial \rho}\right)_{q}=(1+k) \frac{p}{\rho},
$$

which allows to reach Poisson formula $(19)$, recalling that the left-hand side equals $v_{\mathrm{s}}^{2}$.

Poisson did not measure experimentally $\alpha_{q}$ to obtain $k$ and $v_{\mathrm{s}}$. He used instead the value of $k$ that he obtained from the experimental value of the speed of sound to get $\alpha_{p}$. The speed of sound used by Poisson was that determined by the members of the French Academy of Sciences in $1738, v_{s}=337.18 \mathrm{~m} / \mathrm{s}$ at $6{ }^{\circ} \mathrm{C}$, from which follows the value $k=0.4254$ from formula 19 . He then employed the value $\theta_{0}=1 / 0.00375{ }^{\circ} \mathrm{C}$, which comes from the Gay-Lussac measurements, to find $\alpha_{q}=1 / 116$ per degree centigrade at $6{ }^{\circ} \mathrm{C}$ from equation 20 .

Comparing equations (23) and (16), we see that the ratio $\gamma$ of the specific heats is related to the ratio $k$ of thermal expansions by $\gamma=1+k$. Taking into account that the ratio of the compressibilities equals $\gamma$, as we have seen above, we reach the identity

$$
\frac{c_{p}}{c_{v}}=\frac{\kappa_{\theta}}{\kappa_{q}}=1+\frac{\alpha_{p}}{\alpha_{q}} .
$$

A relation between the three ratios, which is equivalent to (27), was derived by Poisson in his paper of 1823 on the speed of sound 16. It is worth mentioning that relation (27), derived within the caloric theory, remains valid in thermodynamics.

Assuming that $\gamma=1+k$ is constant, Poisson integrates equation 26 to find that $p \rho^{-\gamma}$ is constant along an 
adiabatic process, a result reported in another publication of 1823 44. This result is equivalent to

$$
p v^{\gamma}=K
$$

a constant along an adiabatic process. Poisson concludes that $\left(\theta_{0}+\theta\right) v^{\gamma-1}$ is also constant.

\subsection{Clément and Desormes experiment}

To test his theory of sound, Laplace needed to know the experimental value of $\gamma$, that is, he needed to know the experimental values of $c_{p}$ and $c_{v}$ to find $\gamma=c_{p} / c_{v}$. As we have commented before, the measurement of the specific heat at constant volume offered a difficulty that was not overcome before the 1880s, spoiling the determination of $\gamma$ from its definition. An alternative form of finding $\gamma$ was provided by experiments carried out by Clément and Desormes, reported in their memoir submitted to the prize of the French Academy of Science for the year 1812. The negative result of the judgment left them dissatisfied and undermined their confidence in their results but not enough to prevent them from publishing the memoir, which occurred later in 1819 [45].

Clément and Desormes experiment is described as follows. A balloon was equipped with a tap and communicated with a barometer intended to measure the pressure inside the balloon, as shown in Figure 4 Initially, the air inside the balloon is rarefied by the use of a pneumatic machine. At this state, the inside pressure is $p_{1}$ smaller than the outside pressure $p_{0}$ and the temperature is the room temperature. The tap is opened, the outside air rushes into the balloon, the temperature rises above the room temperature and the pressure increases. When the inside pressure reaches the outside pressure $p_{0}$ the tap is closed. With the tap closed, the gas cools, reaching the room temperature, and the pressure decreases to a final value $p_{2}$, which is larger than the initial pressure $p_{1}$.

The whole process of the Clément and Desormes experiment can be better understood by the help of the $p-v$ diagram of Figure 3 in which $p$ is the pressure and $v$ is understood as the volume per unit mass of the air inside the balloon. The process $\mathrm{AB}$ from the initial state A to the intermediate state B, when the tap is closed, is understood as an adiabatic process and the variation in pressure is $p_{0}-p_{1}$. From the intermediate state B until the final state $\mathrm{C}$, the gas cools down and $\mathrm{BC}$ is a volume constant process because the tap is closed. The final state $\mathrm{C}$ and the initial state $\mathrm{A}$ have the same temperature and the variation in pressure is $p_{2}-p_{1}$. In an experiment carried out when the room temperature was $12.5^{\circ} \mathrm{C}$ and the pressure was $766.5 \mathrm{~mm} \mathrm{Hg}$, they found $p_{0}-p_{1}=13.81$ and $p_{0}-p_{2}=3.611 \mathrm{~mm} \mathrm{Hg}$ [45].

To estimate $\gamma$, we apply equation $(28)$ to the adiabatic process $\mathrm{AB}$ in Figure 3 to obtain $p_{0} / p_{1}=\left(v_{1} / v_{0}\right)^{\gamma}$. Taking into account that $\mathrm{AC}$ is an isotherm it follows by the Boyle law that $p_{2} / p_{1}=v_{1} / v_{0}$. Therefore, $p_{0} / p_{1}=$

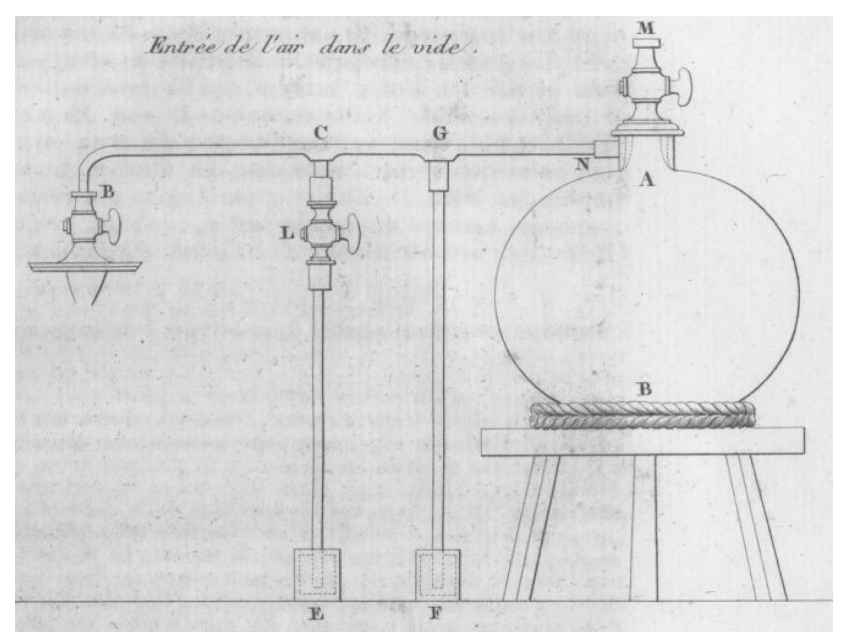

Figure 4: Apparatus used by Clément and Desormes in their experiment reported in 1819 [45]. AB is a glass flask with a capacity of $28.4 \ell$ with a tap $M$ having an aperture of about 1.4 $\mathrm{cm}$ in diameter. The glass flask communicates to a pneumatic machine through a tube NB. This tube also communicated with two other tubes GF and CE, the first of which was immersed in mercury and the second in water. The height of the water in tube CE gives a measure of the pressure inside the flask as the $\operatorname{tap} L$ is open with the taps $B$ and $M$ closed. Figure from reference 45 .

$\left(p_{2} / p_{1}\right)^{\gamma}$, which is written in the form

$$
\gamma=\frac{\ln p_{0}-\ln p_{1}}{\ln p_{2}-\ln p_{1}},
$$

and $\gamma$ could be determined from the measurements of the pressures $p_{0}, p_{1}$, and $p_{2}$. If the differences $p_{0}-p_{1}$ and $p_{2}-p_{1}$ are small compared to $p_{0}$, this relation reduces to

$$
\gamma=\frac{p_{0}-p_{1}}{p_{2}-p_{1}}
$$

The experiment of Clément and Desormes was not intend to determine the ratio of the specific heats, but Laplace used their results to obtain $\gamma=1.3541$ by the use of formula (30) 24. Laplace also used the experimental results obtained by Gay-Lussac and Welter who employed a method similar to that of Clément and Desormes, but they compressed the air inside the balloon instead of rarefying it 24. The two values of $\gamma$ are shown in Table 3 together with the speed of sound determined by equation 12 . The method of Clément and Desormes by compression was also used by Masson [46, 47], by Weisbach [48, and by Cazin 49 to get better values of $\gamma$ for the air, shown in Table 3. We point out that the method of Clément and Desormes in fact measures the ratio $\kappa_{\theta} / \kappa_{q}$ between the compressibilities, which equals the ratio $\gamma=c_{p} / c_{v}$ of the specific heats, as we have seen above.

Cazin [49] used a method in which he combined the two ways of carrying out the Clément and Desormes method, by compression and by expansion. Two vessels of the same capacity contain the same type of gas at 
Table 3: Experimental values of the ratio $\gamma$ of specific heats, and the velocity of sound $v_{\mathrm{s}}$ in $\mathrm{m} / \mathrm{s}$ of the atmospheric air at the temperature $\theta$ in degrees centigrade. CD, Clément and Desoremes; GLW, Gay-Lussac and Welter; MVB, Moll and Van Beek; BM, Bravais and Martins. The second column gives the year of publication of $\gamma$.

\begin{tabular}{|c|c|c|c|c|}
\hline author & year & $\gamma$ & $v_{\mathrm{S}}$ & $\theta$ \\
\hline CD 45, Laplace 24 & 1822 & 1.354 & 332.9 & 12.5 \\
\hline GLW, Laplace 24 & 1822 & 1.37244 & 335.2 & 13 \\
\hline Masson 46,47 & 1847 & 1.4196 & & \\
\hline Weisbach 48 & 1859 & 1.4025 & & \\
\hline Cazin $49-$ & 1862 & 1.408 & & \\
\hline Dulong $3 \overline{6}$ & 1829 & 1.421 & 333 & 0 \\
\hline MVB $51, \overline{\text { Simons } 50}$ & 1830 & 1.4152 & 332.244 & 0 \\
\hline MVB 51, Rankine 29]. & 1850 & 1.40853 & 332.25 & 0 \\
\hline BM 52, Rankine 29 & 1850 & 1.40955 & 332.37 & 0 \\
\hline D \& $\overline{\mathrm{B}} 32$, Joule 58 & 1847 & 1.347 & 333.8 & 0 \\
\hline Regnault 28, Rankine 59 & 1853 & 1.4094 & 332,35 & 0 \\
\hline Regnault $\overline{28}$, Le Conte 60 & 1864 & 1.41047 & 332.490 & 0 \\
\hline
\end{tabular}

different pressures and at the same temperature, and they are connected by a pipe containing a tap. The tap is opened resulting in an equalization of the pressures; one gas warms up and the other cools down. The gases follows the adiabatic processes $\mathrm{AB}$ and $\mathrm{A}^{\prime} \mathrm{B}^{\prime}$ of Figure 5. After closing the tap, the temperatures equalize; and the pressures of the gases become distinct. The gases follow the constant volume processes $\mathrm{BC}$ and $\mathrm{B}^{\prime} \mathrm{C}$. The measurements of the pressures gives the value of $\gamma$ by means of formula 30 . The results of Cazin for the air and other gases are shown in Table 4

\subsection{Ratio $\gamma$ from the speed of sound}

Once Laplace formula (11) is accepted, the ratio $\gamma$ can be determined from the experimental values of the speed of sound. In an experiment carried out by Dulong, reported in 1829 [36], he determined the speed of sound for several gases from the measurement of the frequency of the fundamental mode of vibration and the wavelength of a standing wave in a tube filled with a gas. From the frequency and wavelength, he obtained for the atmospheric air at zero degree centigrade the values of $v_{s}$ and $\gamma$, shown in Table 3 Results of Dulong for other gases are shown in Table 4. Later on, in 1858, Masson 47] used the value of $v_{s}$ that he determined experimentally

Table 4: Ratio $\gamma$ of specific heats for several gases determined by Dulong [36], by Rankine [59], by Masson [47], and by Cazin [49].

\begin{tabular}{|c|c|c|c|c|}
\hline author & Dulong & Rankine & Masson & Cazin \\
\hline year & 1829 & 1853 & 1853 & 1862 \\
\hline air & 1.421 & 1.4094 & 1.4148 & 1.408 \\
\hline oxygen & 1.415 & 1.4014 & 1.4148 & 1.408 \\
\hline nitrogen & & & 1.4148 & 1.408 \\
\hline hydrogen & 1.407 & 1.4150 & 1.4148 & 1.408 \\
\hline carbon monoxide & 1.428 & 1.4047 & 1.4248 & 1.408 \\
\hline nitric oxide & & & 1.4049 & \\
\hline hydrogen chloride & & & 1.4084 & \\
\hline carbon dioxide & 1.348 & 1.2714 & 1.2886 & 1.291 \\
\hline nitrous oxide & 1.343 & & 1.2813 & 1.285 \\
\hline sulfur dioxide & & & 1.2619 & 1.262 \\
\hline ammonia & & & 1.3146 & 1.328 \\
\hline ethylene & 1.240 & & 1.2708 & 1.257 \\
\hline
\end{tabular}

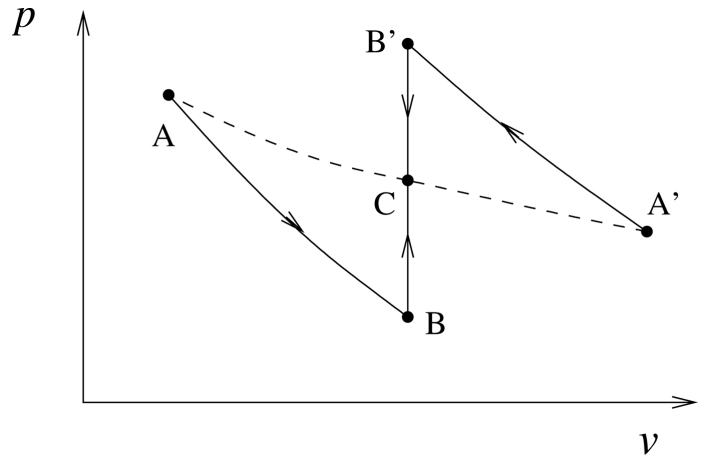

Figure 5: Representation in the $p-v$ diagram of a processes followed by the gases contained in two connected vessels. One gas follows the process $A B C$ and the other the process $A^{\prime} B^{\prime} C$. The processes $A B$ and $A^{\prime} B^{\prime}$ are adiabatic. The processes $B C$ and $B^{\prime} C$ are processes at constant volume. At states $A, C$, and $A^{\prime}$ the gases have the same temperature, that is, the dashed line is an isotherm.

to get more precise values of $\gamma$ for the air and other gases, as shown in Table 4 The method used by Masson was similar to that of Dulong and was carried out by resonating a copper or tin organ pipe placed in a glass balloon of $25 \ell$.

Values of $\gamma$ for the air were also determined by Simons [50] and by Rankine [29], shown in Table 3 by the use of Laplace formula and from the speed of sound obtained by other authors 51,52 by a direct method, which consists in the observation of the flashes of firearm shots between two stations separated by a large distance.

\section{Thermodynamics}

\subsection{Mayer and Joule}

Mayer in 1842 [53 and Joule in 1843 [54] independently formulated the fundamental law connecting heat and work. According to this law the dissipation of work causes the appearance of heat and that a certain amount of work $w$ yields the same quantity of heat $q$. The Mayer-Joule law is represented by the relation $w=J q$, where $J$ is a 
universal constant, called the mechanical equivalent of heat. The same relation is valid when heat is transformed into work. Subsequently, the Mayer-Joule law evolved into or was understood as the law of conservation of energy.

Joule used various methods to determine the mechanical equivalent of heat such as the well known paddle-wheel method [55]. In 1845 [56], he reported an experiment in which the mechanical equivalent of heat was determined by the compression of air. The apparatus he used is shown in Figure 6. Dry air was compressed in a cooper receiver immersed in water by the use of a pump. By measuring the rise in the temperature of the water, he could determine the quantity of heat $q$ evolved by compression of air. In one series of experiments, carried out with 300 strokes of the pump each, $2956=v_{1}$ cubic inches of air at the pressure of $30.2=p_{1}$ inches of mercury is compressed into the receiver of $136.5=v_{2}$ cubic inches. Assuming an isothermal compression the work performed by the pump is $w=p_{1} v_{1} \ln \left(v_{1} / v_{2}\right)$, a result that is obtained from the equation of state of an ideal gas, Joule finds $w$ to be equivalent to raise 11220.2 pounds by one foot. From the rise in temperature of the water, it is found that $q$ is the heat equivalent to increase by one degree Fahrenheit 13.628 pounds of water. From the ratio of the last two numbers, Joule finds $J=w / q$ to be equivalent to raise one pound by 823 feet. In modern units, this corresponds to $4.43 \mathrm{~J} / \mathrm{cal}$.

According to Joule his experimental results were in perfect accordance with Dulong law (4) [56]. However, taken into account that his experiment corresponds to an isothermal compression, it seems that he interpreted law (4) in the same sense of Clapeyron, that is, as equivalent to law (5).

In the same paper of 1845 [56], Joule also reported experiments on the free expansion of air. He connected two equal cooper vessels by a coupling nut with a stopcock, as shown in Figure 7. One of the vessels was filled with 22 atm of dry air, the other was exhausted, and the two were immersed in water. The stopcock was then opened allowing the passage of air from one to the other vessel. The temperature of the water was measured before and after the expansion but no change of temperature was observed. He concluded that

(7) No change of temperature occurs when air is allowed to expand in such a manner as not to develop mechanical power.

That is, in a free expansion of air, the temperature remains constant.

The method conceived by Mayer to determine the mechanical equivalent of heat $J$ was distinct from that of Joule and needed the experimental value of the ratio of specific heats. He explained his method in a publication of 1845 [57. When a gas is expanded at a constant pressure $p$, it consumes a certain amount of heat $q_{p}$ and performs a work $p \Delta v$ where $\Delta v$ is the increase in the volume of

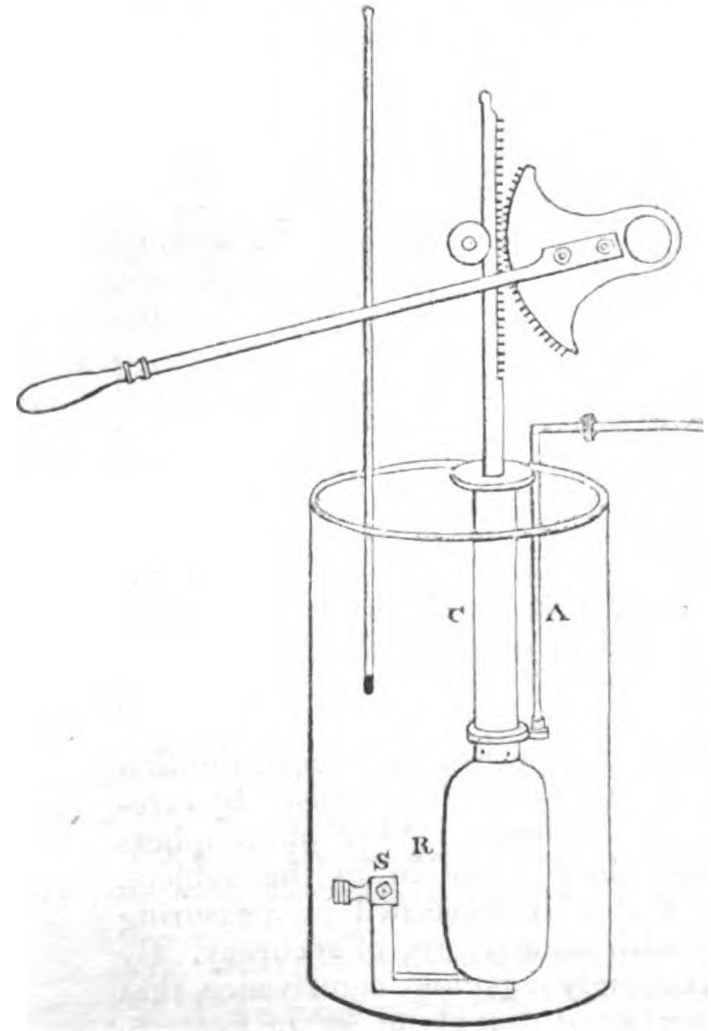

Figure 6: Apparatus of Joule to measure the heat evolved by compression of the air [56]. The pump $C$ and the cooper receiver $\mathrm{R}$ are immersed in water contained in a vessel impermeable to heat, in which there is a sensible thermometer. The pump is operated several times, compressing dry air, which is admitted through the pipe $A$, until the air inside the receiver reaches about twenty two atmospheres, generating a certain quantity of heat which warms the water. Figure from reference [56].

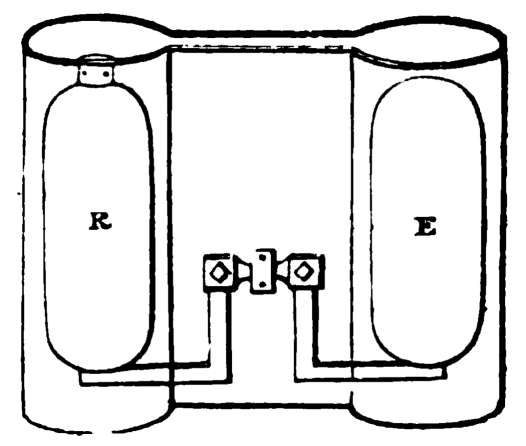

Figure 7: Joule free expansion apparatus [56]. $\mathrm{R}$ and $\mathrm{E}$ are two cooper receivers with capacities of 136.5 and 134 cubic inches. The two receivers are connect by a pipe with a stopcock and immersed in water contained in a vessel impermeable to heat. Figure from reference [56].

the gas. Mayer argues that part of the heat consumed in this isobaric process is transformed into work performed by the gas and the other part, which is retained by the gas, is equivalent to the heat $q_{v}$ that would be consumed by the gas if its volume was kept constant. Using the equivalence between heat and work, these reasonings lead 
to the relation

$$
J\left(q_{p}-q_{v}\right)=p \Delta v .
$$

For an ideal gas, $p \Delta v=r \Delta \theta$, at constant pressure, and dividing relation (31) by the increase in temperature $\Delta \theta$, Mayer finds the relation 57

$$
J\left(c_{p}-c_{v}\right)=r .
$$

He applied the relation to the atmospheric air by computing $r=p / \rho T$ from the density of air at $0^{\circ}$ and 1 atm. Mayer used the value of $c_{p}=0.2669 \mathrm{cal} / \mathrm{g}^{\circ} \mathrm{C}$ determined experimentally by Delaroche and Bérard 32], and $\gamma=1.421$ determined experimentally by Dulong [36] to get $c_{v}=c_{p} / \gamma$. To heat one kilogram of water by one degree centigrade he found that it was equivalent to the fall of the same quantity of water by a height of 365 meters, which in modern units is equivalent to $3.58 \mathrm{~J} / \mathrm{cal}$.

Instead of using $\gamma$ to determine $J$ by means of equation (32), as did Mayer, one could as well use $J$, obtained by another method, to find $\gamma$. In fact, this was the method used by Joule in 184758 to obtain $\gamma$ for the atmospheric air. He determined $r=p / \rho T$ from experimental results and, for the mechanical equivalent of heat $J$, he used a value that he had determined previously. This value, which corresponds to the heat necessary to increase a pound of water by one degree Fahrenheit, was equivalent to raise one pound by 775 feet, which in modern units is equivalent to $4.17 \mathrm{~J} /$ cal. He determined $r / J$ and used the value of $c_{p}$ determined experimentally by Delaroche and Bérard 32], $c_{p}=0.2669$, from which he found $c_{v}=0.1977$ by using result (32). From these specific heats, he finds for the air at 32 degrees Fahrenheit the value $\gamma=1.347$. From $\gamma$ he determined the velocity of sound by the use of Laplace formula, results shown in Table 3 The result for $\gamma$ was not so good due to the imprecise value of $c_{p}$ determined by Delaroche and Bérard. The same scheme was used later on by Rankine [59] and by Le Conte [60], with a more precise value of $c_{p}=0.2379$, determined by Regnault 28], which led to the values shown in Table 3 . Rankine also determined $\gamma$, shown in Table 4, for other gases by the same method and by using values of $c_{p}$ determined by Regnault 28.

\subsection{Clausius}

The conservation of energy in differential form,

$$
d u=J d q-d w,
$$

is one of the principles of the mechanical theory of heat, or thermodynamics, that emerged in the middle of the nineteenth century 6 . In contrast to the caloric theory, heat is not a state function, which is equivalent to say that $d q$ is not an exact differential. Likewise, $d w$ is not an exact differential. On the other hand, $d u$ is an exact differential, and is the expression of the conservation of energy. Another principle of thermodynamics, established by Clausius, is 6,30 .

$$
d q / T \text { is an exact differential, }
$$

where $T=\theta_{0}+\theta$ is the absolute temperature. In his first paper on heat, Clausius showed that $J d q-d w$ is an exact differential for an ideal gas through the use of the following law, valid for an ideal gas [21]:

(8) When an ideal gas expands isothermally, all the heat absorbed is converted into work performed by the gas,

which, according to Clausius, is experimentally verified as much as the ideal gas equation 21.

Once the conservation of energy in the form (33) is introduced as a principle of thermodynamics, valid for any system, and together with expression (34), one may derive law (8) and other laws concerning the ideal gas.

For a gas, $d w=p d v$, which replaced in equation (33) yields $d q / T=(d u+p d v) / J T$, which being an exact differential allows us to write

$$
\left(\frac{\partial(1 / T)}{\partial v}\right)_{u}=\left(\frac{\partial(p / T)}{\partial u}\right)_{v} .
$$

For and ideal gas, the right-hand side of this equation vanishes identically because $p / T$ depends only on $v$, and we may conclude that $(\partial T / \partial v)_{u}=0$, which is the expression of law (7) if we bear in mind that in the free expansion of a gas in the Joule experiment the energy remains constant. This relation is equivalent to $(\partial u / \partial v)_{T}=0$, which states that

(9) The internal energy of an ideal gas depends on the temperature but not on the volume.

From law (9), we see that in an isothermal expansion the energy $u$ remains constant so that $J q=w$ which is the mathematical expression of law (8). The heat absorbed by an ideal gas along an isothermal process can thus be determined by calculating the work performed by the gas in this process. Along an isotherm between two states with volumes $v_{1}$ and $v_{0}$ the work is $r T \ln \left(v_{1} / v_{0}\right)$ so that the heat absorbed $q_{1}$ is

$$
J q_{1}=r T \ln \left(v_{1} / v_{0}\right)=p v_{0} \ln \left(v_{1} / v_{0}\right),
$$

which expresses Carnot law (5) because the right-hand side does not depend on the nature of the gas.

Let us determine the heat related to law (4), understood as the heat $q_{2}$ released along the process ABC shown in Figure 3 which can be determined by calculating the work along the adiabatic process BA. Assuming that $\gamma$ is constant, we use Poisson result that $p v^{\gamma}$ is constant along the adiabatic process to get

$$
J q_{2}=\frac{p v_{0}}{k}\left(1-\left(v_{1} / v_{0}\right)^{k}\right),
$$


where $k=\gamma-1$. We see that the heat is not the same for all ideal gases, which invalidate law (4), within thermodynamics. However, if the difference $v_{1}-v_{0}$ is small one obtains $J q_{2}=J q_{1}=p\left(v_{1}-v_{2}\right)$, in which case law (4) becomes valid and also equivalent to law (5).

Let us consider now the specific heats of an ideal gas. From $J d q=d u+p d v$ we find

$$
J c_{p}=\left(\frac{\partial u}{\partial T}\right)_{p}+p\left(\frac{\partial v}{\partial T}\right)_{p}
$$

and

$$
J c_{v}=\left(\frac{\partial u}{\partial T}\right)_{v} .
$$

For an ideal gas, $u$ depends only on the temperature so that the first term on the right-hand side of 38 equals the right-hand side of (39). In addition, for an ideal gas, the second term on the right-hand side of 38 equals $r$, and we conclude that

$$
J\left(c_{p}-c_{v}\right)=r
$$

Multiplying this relation by the density $\rho$ one finds

$$
J\left(\bar{c}_{p}-\bar{c}_{v}\right)=p / T
$$

which is an expression of the Carnot law (6).

The two relations 40 and 41 were derived by Clausius 21] in a manner distinct from that we have done here. Relation (40) was also derived by Mayer, as we have seen above. The reasoning used by Mayer is equivalent to say that $(\partial u / \partial T) p$ equals $J c_{v}$, which is not in general correct. However, as stated above, this equality becomes valid for an ideal gas, making the Mayer procedure justifiable in this case.

Taking into account that $u$ depends only on the temperature, the same happens to $c_{v}$, and in view of relation 40 it also happens to $c_{p}$, a result that is equivalent to Regnault law (2).

\section{Avogadro law}

The ideal gas also played a relevant role in the development of the molecular theory of matter, specially in determination of molecular weights and establishment of chemical formulas. In 1809, Gay-Lussac announced the law of combining volumes of gases. According to this law, the volumes of the combining gaseous substances and the volume of resulting gas substance are proportional to small integer numbers [61]. For example, the volumes of the combination of hydrogen with oxygen to produce water vapor are in proportion to the numbers, 2, 1 and 2, in which case there is a contraction of the total volume. After the combination, the conditions, that is, pressure and temperature, should be the same as before. This law was taken over by Berzelius as the basis of his theory of volumes for chemical composition. It helped Berzelius do adopt the chemical formula $\mathrm{H}_{2} \mathrm{O}$ for water, which was interpreted by Berzelius as 2 volumes of hydrogen combine with 1 volume of oxygen to produce water [62]. This interpretation is distinct from that given by Dalton atomic theory $[63$ in two ways. In Dalton theory, the symbols $\mathrm{H}$ and $\mathrm{O}$ represent the atoms, and not volumes, of hydrogen and oxygen, and the atom of water was represented by $\mathrm{HO}$.

In 1811 Avogadro announced a molecular hypothesis [9], called Avogadro hypothesis by Cannizzaro in 1858 [64 and Avogadro law by Naumann in 1869 [65], which could conciliate Berzelius theory of volumes and Dalton atomic theory. To state his law, Avogadro chose the word 'molecule' to represent the smallest part of a pure substance. According to Avogadro law, equal volumes of distinct gases under the same temperature and pressure contain the same number of molecules 66. Three years later, Ampère published an article containing ideas similar to those of Avogadro, using the word 'particle' in the place of 'molecule' 67]. In 1826 [68], Dumas determined experimentally the atomic weight of several substances from their densities at the gaseous state. To this end he used a rule stating that, at the same pressure and temperature, the molecules of all gases are placed at equal distance, which we may understand as a consequence of Avogadro law if we interpret equal distance on the average. Similar ideas were proposed by Gaudin in a paper of 1833 [69] in which he clearly distinguished atoms and molecules, and proposed that the molecules could be composed of one or more atoms, of the same kind or distinct, which he called monatomic, diatomic, triatomic, and so on. He proposed that some simple gases are diatomic and illustrated his ideas by the use of volume diagrams as those shown in Figure 8. Gaudin did not mention Avogadro and his theory received little scientific recognition.

Avogadro law allows us to replace 'volume' by 'number of molecules' in the Gay-Lussac law of combining volumes. For water it amounts to say that 2 molecules of hydrogen combines with 1 molecule of oxygen to produce 2 molecules of water, which according to Berzelius is represented by $2 \mathrm{H}+\mathrm{O} \rightarrow 2 \mathrm{H}_{2} \mathrm{O}$. But in this representation wee see that the total mass is not conserved. To solve this problem, Avogadro makes the assumption that the molecule of hydrogen and oxygen are composed by two elementary molecules of the same type, and now $2 \mathrm{H}_{2}+\mathrm{O}_{2} \rightarrow 2 \mathrm{H}_{2} \mathrm{O}$, and the total mass is conserved. Thus, Avogadro theory for atomic weights is not only based on his law but also on the assumption concerning the constitution of molecules of simple gases by elementary molecules of the same species. It should be remarked that Avogadro did not make use of chemical symbols in his paper of 1811 .

In spite of likely solving the existing inconsistencies of the atomic theory, Avogadro law was neglected or misunderstood by the majority of the contemporary chemists for almost five decades [9]. Even when his law was mentioned, sometimes it was attributed to Ampère, some- 


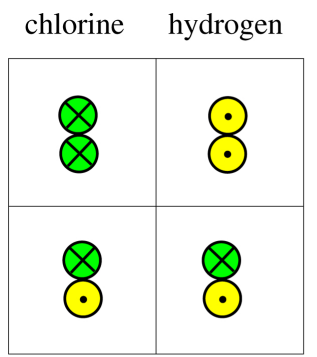

hydrogen chloride

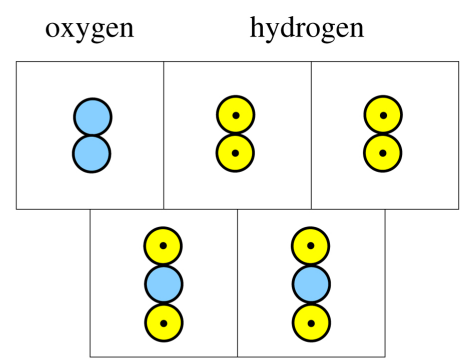

water vapor

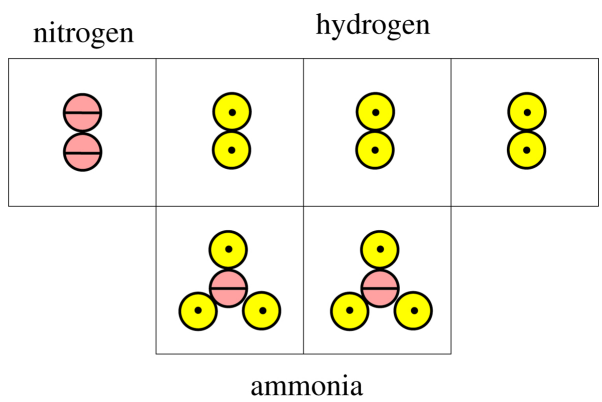

ammonia

Figure 8: Volume diagrams used by Gaudin [69]. Each one of the identical volumes (represented by squares) contains the same number of molecules (just one molecule), in accordance with Avogadro law. Left: Chlorine and hydrogen giving hydrogen chloride, $\mathrm{Cl}_{2}+\mathrm{H}_{2} \rightarrow 2 \mathrm{HCl}$. Center: Oxigen and hydrogen given water vapor, $\mathrm{O}_{2}+2 \mathrm{H}_{2} \rightarrow 2 \mathrm{H}_{2} \mathrm{O}$. Right: Nitrogen and hydrogen giving ammonia, $\mathrm{N}_{2}+3 \mathrm{H}_{2} \rightarrow 2 \mathrm{NH}_{3}$. Gaudin used Dalton symbols instead of chemical symbols.

times to Gay-Lussac, but seldom to Avogadro [70]. Finally, Avogadro's theory was revived by Cannizzaro in his paper on atomic weights of 1858 [64]. Influenced by Gaudin 71], he provided a clear distinction between a molecule and an atom which corresponds to the modern understanding of these two concepts. He also interpreted Avogadro's idea concerning the constitution of molecules of some elementary gases as composed by two atoms of the same kind. Cannizzaro remarked that the differentiation between atoms and molecules would clarify the confusing state of affairs concerning atomic weights and chemical composition [9. From 1858 onwards, Avogadro law began to be recognized and accepted by the chemists. For instance, it was adopted as a fundamental law by Meyer and by Mendeleev in their successful textbooks of 1864 and 1868, respectively 9. In the second edition of his textbook 72 73, Meyer states that the Avogadro law together with the law of Dulong and Petit $[39$ are widely recognized as the bases for the determination of atomic and molecular weights. Nernst adopts Avogadro law as a fundamental law of theoretical chemistry as shown up in the title of his book on the subject of 1893 [74.

A support for Cannizzaro interpretation of Avogadro theory came from Clausius who established a connection between Avogadro law and the kinetic theory of gases. He also made a clear differentiation between atoms and molecules, and also admitted that a molecule could be composed of two identical atoms [9]. As a simple example, Clausius considered the nitric oxide, assumed to have the chemical formula NO. One volume of oxygen combines with one volume of nitrogen to give two volumes of nitric oxide. In accordance with Avogadro law, one molecule of oxygen combines with one molecule of nitrogen to give two molecules of nitric oxide, which is possible only if the molecules of nitrogen and oxygen are formed by two equal atoms.

By using Avogadro law, the molecular weight of a gas, which is the mass of a molecule in relation to another taken as standard, is determined by the measurement of its densities. The density of a gas can be written as $\rho=N m / V$ where $m$ is the mass of a molecule and $N$ is the number of molecules in a volume $V$. According to
Avogadro law, the ratio $N / V$ is the same for all gases at the same temperature and pressure leading to conclude that $\rho$ is proportional to $m$ and thus proportional to the molecular weight $\mu$. Therefore, the ratio of the densities $\rho / \rho_{0}$ of two gases at the same temperature and pressure is equal to the ratio $\mu / \mu_{0}$ of the molecular weights, that is, $\mu=\mu_{0} \rho / \rho_{0}$. If we consider $\rho$ as the density of a gas with respect to atmospheric air, as did Avogadro and Cannizzaro, and setting $\mu_{0}=2$ for the hydrogen, then $\mu_{0} / \rho_{0}=28.87$, according to Cannizzaro [64], and the molecular weight is $\mu=28.87 \rho$. The molecular weight of several gases determined by Avogadro and Cannizzaro are shown in Table 5 together with the chemical formulas employed by Cannizzaro.

The equation of state of an ideal gas can be written in a form which explicitly includes the Avogadro law. Taking into account that $\rho=N m / V$, equation (6) is written as $p=(N / V) m r T$. Since according to Avogadro law, the ratio $N / V$ is the same for all gases at the same temperature and pressure, it follows that $m r=k$ is a constant independent of the nature of the gas, and the

Table 5: Column 2: Density $\rho$ of gases in units where that of air is taken as unity, according to Gay-Lussac [26]. Column 3: Molecular weight $\mu^{*}$ of gases with respect to molecular weight of hydrogen taken as unity, according to Avogadro [66]. Column 4 and 5: Molecular weight $\mu$ of gases with respect to molecular weight of hydrogen taken as equal to 2 , and chemical formulas (cf), according to Cannizzaro 64].

\begin{tabular}{lcccc}
\hline gas & $\rho$ & $\mu^{*}$ & $\mu$ & $\mathrm{cf}$ \\
\hline oxygen & 1.10359 & 15.074 & 32 & $\mathrm{O}^{2}$ \\
nitrogen & 0.96913 & 13.238 & 28 & $\mathrm{~N}^{2}$ \\
hydrogen & 0.07321 & 1 & 2 & $\mathrm{H}^{2}$ \\
carbon monoxide & 0.96782 & 13.22 & 28 & $\mathrm{CO}$ \\
nitric oxide & 1.03636 & 14.156 & 30 & $\mathrm{NO}$ \\
chlorine & 2.468 & 33.74 & 71 & $\mathrm{Cl}^{2}$ \\
hydrogen chloride & 1.278 & 17.45 & 36.5 & $\mathrm{HCl}^{2}$ \\
carbon dioxide & 1.5196 & 20.75 & 44 & $\mathrm{CO}^{2}$ \\
nitrous oxide & 1.52092 & 20.775 & 44 & $\mathrm{~N}^{2} \mathrm{O}$ \\
sulfur dioxide & 2.265 & 30.94 & 64 & $\mathrm{SO}^{2}$ \\
ammonia & 0.59438 & 8.119 & 17 & $\mathrm{NH}^{3}$ \\
year & 1809 & 1811 & 1858 & 1858 \\
\hline
\end{tabular}


ideal gas equation reads

$$
p V=N k T,
$$

which incorporates Avogadro law, because $k$ is a universal constant. It should be remarked that this form of the equation of state did not appear before the introduction of the constant $k$ by Planck in his paper of 1900 on energy distribution of radiation 75$]$.

Let $N_{0}=\mu / m$ be the number of molecules of an amount of gas of mass equal to $\mu$ in grams. Since $m r=k$ is a universal constant it follows that $\mu r=R$ is also a universal constant and $R=k N_{0}$. In 1867, Guldberg reached this conclusion, that $\mu r$ is a constant, as a consequence of the "chemical theory of molecular volumes" [76], which should be understood as the Avogadro law. Defining $n=N / N_{0}$, we may write the gas equation as

$$
p V=n R T \text {. }
$$

Next we define $\bar{v}=V / n$, which is the volume of an amount of gas of mass $\mu$, and equation (43) becomes

$$
p \bar{v}=R T .
$$

In this form, the ideal gas equation was written by Horstmann ${ }^{2}$ in 1873 77.

Ostwald, in his book on physical chemical measurements of 1893 [78, called a mole ( Mol in German) the number of molecules in an amount of gas of mass $\mu$, which is $N_{0}$. He also determined the gas constant and found ${ }^{3}$ $R=8.308 \mathrm{~J} / \mathrm{mol}^{\circ} \mathrm{C}$. The volume $\bar{v}$ of an amount of gas of mass $\mu$ is also the volume of one mole of the gas and $n$ in equation (43) is identified as the number of moles. In his book on theoretical chemistry of 1893 [4], Nernst wrote both forms (44) and (43) for an ideal gas and used equation (44) to determine $R$. The value of the molar volume $\bar{v}=\mu / \rho$ was obtained from experimental data for the densities of hydrogen, oxygen and nitrogen and from their molecular weights. The average from these three gases was $\bar{v}=22.42 \ell / \mathrm{mol}$ at $0{ }^{\circ} \mathrm{C}$ and $1 \mathrm{~atm}$, which yields the value $R=8.321 \mathrm{~J} / \mathrm{mol}^{\circ} \mathrm{C}$.

The discovery of argon by Rayleigh and Ramsay in 1894 is directed related to Avogadro law. Rayleigh noticed a discrepancy between the density of the nitrogen obtained from the atmospheric air and the nitrogen obtained from nitrogen compounds. The densities of the former and the latter were found to be 1.2834 and 1.2774 , respectively, which gives a ratio 1.0047 between the molecular weights of the atmospheric nitrogen and the nitrogen. The discrepancy, however small, was sufficient to indicate the presence of impurity in the atmospheric nitrogen, which lead to the discovery of argon 79 .

\footnotetext{
${ }^{2}$ Horstmann wrote $p u=R T$ using the letter $u$ for the volume. ${ }^{3}$ The value given by Ostwald was 84720 in units in which the pressure is measured in $\mathrm{g} / \mathrm{cm}^{2}$, and the volume in $\mathrm{cm}^{3}$. This figure should be multiplied by the acceleration due to gravity to obtain the value above.
}

\section{Equipartition of energy}

\subsection{Translational equipartition}

The kinetic theory, which emerged around the middle of the nineteenth century, aimed to derive the macroscopic properties of bodies from the behavior of its microscopic constituents. The constituents, which are molecules in the case of gases, were assumed to follow the Newtonian mechanics. The most simple object of study was an ideal gas, that is, a gas at low densities so that the average distance between molecules is large. This property results in a small interaction between molecules so that the total energy of the gas is just the sum of the kinetic energy of each molecule. The first challenge of the theory was the derivation of the known equation of state of an ideal gas.

The kinetic model for an ideal gas consists of molecules that move in straight lines in any possible direction, striking with each other and with the walls of the container. This model was proposed independently by Bernoulli in 1738 , by Herapath in 1821, and by Waterston in 1845 , and was pursued by Joule in 1848 , but it began to thrive only after 1857 when Clausius published a paper entitled "On the kind of motion which we call heat" 80. The publication of his paper was stimulated by a publication by Krönig on the same subject in 1856 [81]. However, Clausius's conceptions about the nature of the motion called heat arose before 1850 [80], and led him to an independent and more thorough proposal of the kinetic model.

Clausius assumes that the molecules moves with the same speed $c$ in arbitrary directions, and arrives at the following relation between the pressure $p$ of the gas and the speed $c$ of the molecules

$$
p V=\frac{1}{3} N m c^{2},
$$

where $V$ is the volume of the vessel, $N$ is the number of molecules and $m$ is the mass of each molecule. This relation states that $p V$ is $2 / 3$ of the translational kinetic energy of molecules.

Clausius reasonings was as follows. Consider a cubic vessel of edge length $L$ and let $v_{x}, v_{y}$ and $v_{z}$ be the Cartesian components of the velocity of a molecule. When a molecule collides with a wall perpendicular to the $z$ axis the change in the momentum of the molecule is $2 m v_{z}$. The time it takes for the next collision on the same wall is $L / v_{z}$ so that the force is $2 m v_{z}^{2} / L$ which is written as $\left(2 m c^{2} / L\right) \cos ^{2} \beta$ where $\beta$ is the angle between the velocity and the $z$ axis. Since the direction of velocity is arbitrary we take the average of this force over all angles such that $\beta \geq \pi / 2$. The average of $\cos ^{2} \beta$ is

$$
\int_{0}^{\pi / 2} \cos ^{2} \beta \sin \beta d \beta=\frac{1}{6},
$$

and the average force becomes $m c^{2} / 3 L$. For $N$ molecules the force is $N m c^{2} / 3 L$ and as the pressure $p$ is this force 
divided by the area $L^{2}$ of a face of the cubic vessel, one finds $p=N m c^{2} / 3 V$ which is the result 45).

In his paper on the kinetic theory, Waterston also arrived at the result (45) but he missed the factor $1 / 3[82$. In the same paper, he announced the law that in mixed media the mean square molecular velocity is inversely proportional to the specific weight of the molecules [82], which was considered by Rayleigh as the first statement of the equipartition of energy for translational motion. The paper was submitted in 1845 to the Royal Society of London but it remained unnoticed until it was found by Rayleigh who published the paper in 1893 [82. However, the law appeared in a short communication published in 1852 [83] ensuring to Waterston the priority of the law, concerning the translational motion [84].

The derivation of the equipartition law carried out by Waterston is far from being satisfactory 84 and cannot be considered as a logical derivation from the assumptions of the kinetic model, what does not mean that the conclusion is necessarily incorrect. Additionally, Waterston shows that the equipartition holds if the gases have the same pressure and the same value of $N / V$ 82], which can be understood as the Avogadro law.

Clausius also arrived at the equipartition law for translational motion but he did not present any derivation from the assumptions of the kinetic theory. He arrived at the equipartition from Avogadro law, which he admitted to be a very probable assumption, and by using relation 45. In his words, when pressure and temperature are the same and contain the same number of atoms in equal volumes, it follows that the atoms of different gases must have equal translational kinetic energy [80]. It should be remarked that Clausius and Waterston used Avogadro law but they did not mention the name of the Italian physicist.

The translational equipartition can be demonstrated from Avogadro law as follows. We have seen that equation (42) incorporates Avogadro law because $k$ being a universal constant the ratio $V / N$ is the same for all gases at the same temperature and pressure. Comparing equations 42 and 45 we find

$$
\frac{1}{2} m c^{2}=\frac{3}{2} k T
$$

Since $k$ is universal, that is, it does not depend on the gas, we see that the translational kinetic energy of a molecule $m c^{2} / 2$ is the same for all gases at the same temperature and equals $(3 / 2) k T$. We see that $c^{2}$ is inversely proportional to mass of the molecule, which is the way Waterson announced the equipartition, mentioned above.

The kinetic theory of gases conceived by Clausius [80] differed in a fundamental aspect from the previous theories. In addition to the translational motion, the molecules could have other types of motion such as rotation and vibration motions, which Clausius called motions of the constituents. This aspect was essential in the description of the specific heat of gases composed by diatomic molecules. In spite of the additional types of motion, the pressure of a gas is caused by the translational motion only and is given by equation (45). That is, the translational kinetic energy,

$$
K=\frac{1}{2} N m c^{2},
$$

is related to the pressure by $p V=(2 / 3) K$.

Assuming that the specific heat $c_{v}$ of an ideal gas is a constant $c$, Clausius shows that the total kinetic energy $U$ has a constant ratio to the translational kinetic energy $K$, that is,

$$
\frac{K}{U}=\frac{3}{2}(\gamma-1)
$$

Indeed, the total energy can be written as $U=M J c T$ because $c_{v}$ is a constant and $M$ is the mass of the gas. From relation 40, we see that the ratio of the specific heats is $\gamma=1+p V / U$. Taking into account the relation $p V=(2 / 3) K$, one reaches equation 49$)$. This relation can be understood as a partition of the energy but not the equipartition of the energy. Clausius applied this relation to "simple gases, and compound gases that suffer no decrease in volume in the combination", which are identified as diatomic gases. For these gases $\gamma=1.421$ and $K / U=0.6315$.

\subsection{Maxwell equipartition of energy}

In his first paper on kinetic theory of 1860 85], Maxwell considers a gas model which was an improvement on Clausius model. In Maxwell's model, the particles, which are perfectly elastic spheres, move with distinct speeds in arbitrary directions. In the first part of the paper he consider the particles to be perfectly elastic spheres. According to Maxwell the distribution of the velocities is the same as that of errors in measurements. The idea of using statistics in physics was not knew since Laplace and Gauss employed it in the theory of errors, but Maxwell used statistics to describe physical processes themselves, which turned out to be an original idea and "marks the beginning of a new epoch in physical sciences" [86]. In this sense, the introduction of statistical approach in physics is due to Maxwell [87].

Denoting by $v_{x}, v_{y}$ and $v_{z}$ the Cartesian coordinates of the velocity, the Maxwell probability distribution of these variables is

$$
\rho\left(v_{x}, v_{y}, v_{z}\right)=b e^{-\left(v_{x}^{2}+v_{y}^{2}+v_{z}^{2}\right) / a},
$$

where $a$ is a constant and $b=(a \pi)^{-3 / 2}$ is the normalization factor. From equation (50), Maxwell derives the distribution of the speeds $v=\left(v_{x}^{2}+v_{y}^{2}+v_{z}^{2}\right)^{1 / 2}$,

$$
\rho_{\mathrm{s}}(v)=4 \pi b v^{2} e^{-v^{2} / a}
$$

It should be mentioned that the derivation of the distribution of velocities provided by Maxwell in his paper of 1860 cannot be considered as a valid derivation from 
pure mechanics 88. Lacking a proper derivation, the distribution may be introduced as a postulate of the kinetic theory of gases, that is, a law that is considered to be true without the need of demonstration.

Maxwell showed that equation 45 is also valid within his kinetic theory. It suffices to interpret $c^{2}$ as as the average of the square of the velocity of a particle, $c^{2}=$ $\left\langle v^{2}\right\rangle$.

In the second part of his first paper on kinetic theory 85, Maxwell also considers a gas consisting of two types of particles and reaches the equipartition of translational energy in the form

$$
\frac{1}{2} m_{1} c_{1}^{2}=\frac{1}{2} m_{2} c_{2}^{2}
$$

where $m_{i}$ is the mass and $c_{i}^{2}=\left\langle v_{i}^{2}\right\rangle$ is the mean square velocity of particles of type $i$. A molecule with greater mass moves on the average slower than one with a smaller mass. Form 52 of equipartition is equivalent to the translational equipartition that we have considered above, in which two distinct gases are at the same temperatures. From the translational equipartition, Maxwell reaches the Avogadro law, which he calls chemical law, but does not mention the name of the Italian physicist 85. In other papers 89 91, Maxwell states that it is a relevant law, known as law of equivalent volumes, and a necessary and important consequence of the the kinetic theory of gases, although he incorrectly says that the law was established by Gay-Lussac from chemical considerations. Avogadro law was also derived from the translational equipartition by Naumann in a publication of 1869 65.

In the third part of the first paper on the kinetic theory 85], Maxwell considers the case of particles that are perfectly elastic bodies of any form. In this case, in addition to translational motion, the particles may have rotational motion. Here, Maxwell reaches an equipartition of energy with respect to translational as well as to rotational motion, that is,

$$
\begin{aligned}
& \frac{1}{2} m\left\langle v_{x}^{2}\right\rangle=\frac{1}{2} m\left\langle v_{y}^{2}\right\rangle=\frac{1}{2} m\left\langle v_{z}^{2}\right\rangle= \\
& =\frac{1}{2} I_{1}\left\langle\omega_{1}^{2}\right\rangle=\frac{1}{2} I_{2}\left\langle\omega_{2}^{2}\right\rangle=\frac{1}{2} I_{3}\left\langle\omega_{3}^{2}\right\rangle,
\end{aligned}
$$

where $I_{1}, I_{2}, I_{3}$ and $\omega_{1}, \omega_{1}, \omega_{2}$ and $\omega_{3}$ are the moments of inertia and angular velocities around the principal axes of the body.

The equipartition of energy is sometimes referred to as a theorem, that is, a proposition that is a logical derivation from some given premises, which in the present case are the laws of motion of Newtonian mechanics. In this sense, the derivation presented by Maxwell in the paper of 1860 [85] of the equipartition of energy as given by equation (52) or by equation (53) is unsatisfactory 82 and cannot be considered as valid derivation from pure mechanics 92 . The demonstrations of the equipartition of energy that came latter by Maxwell in 1867 [89] and 1879 [93], by Boltzmann in 1868 [94 and 1871 [95], and by Watson in 1877 , w6 were based on the use probabilistic approach to kinetic theory, and cannot either be considered as demonstrations from pure mechanics.

In his paper on thermal equilibrium of polyatomic gases of 1871 [95], Boltzmann states that a consequence of the translational equipartition is that all gases at the same pressure and temperature have the same number of particles per unit volume, a result which is confirmed with experience. This is the Avogadro law, but he did not mention Avogadro's name.

From the results given by equation (53), Maxwell concludes that the translational kinetic energy is half of the total energy, that is, $K / U=1 / 2$. On the other hand, if, following Maxwell, one replaces the experimental value $\gamma=1.408$ in Clausius formula (49), one finds the value $K / U=0.612$. Maxwell concludes his paper of 1860 by stating that the theoretical value $1 / 2$ of $K / U$ does not agree with 0.612 obtained from the experimental value of $\gamma$, but gives no reason for the discrepancy.

The equipartition given by formula $(53)$ was obtained by Maxwell considering a molecule as a rigid body. In 1871, Boltzmann gave a similar result by considering a molecule as consisting $n$ atoms, each one being a material point, showing that the kinetic energy of each atom is the same 95. Using this result, Boltzmann finds for a diatomic gas, $n=2$, the same result found by Maxwell for the ratio between the translational kinetic energy and the total kinetic energy, that is. $K / U=1 / 2$. From Clausius formula $49, \gamma=4 / 3$ which is distinct from the experimental value $\gamma=1.41$ for the air. Like Maxwell, Boltzmann gives no explanation for the discrepancy except a possible interaction with the ether, which did not solve the problem either.

In view of the discrepancy shown by diatomic gases, Kundt and Warburg found it appropriate to study a simpler case, namely, that of the mercury vapor, which was known by the chemists to be a monatomic gas. The value of $\gamma$ for mercury vapor, obtained by Kundt and Warburg in 1875 from the experimental value of the speed of sound, was $\gamma=1.67$ [97]. If this result is replaced in Clausius equation (49) the result is $K / U=1$ which means that the total energy coincides with the translational kinetic energy or, in other terms, the molecules possess only translational motion. Kundt and Warburg concluded that a monatomic molecule acts like a material point 97. However, the problem of diatomic gases remained unsolved.

\subsection{Equipartition and degrees of freedom}

In a lecture delivered at the Chemical Society in 1875 [91, Maxwell presented the following formula relating the ratio $\gamma$ and the number of degrees of freedom $\nu$ of a molecule

$$
\gamma=1+\frac{2}{\nu+e}
$$

where $e$ is a term related to the vibration of the molecule, which takes the value $e=0$ when the molecule is rigid. 
Formula (54) was also obtained by Watson in 1876 96 and Boltzmann [98]. To reach this relation, Maxwell consider that the kinetic energy related to each degree of freedom is the same, which we denote by $a$. The energy $u$ of a molecule is the sum of its kinetic enery $\nu a$ plus a term $\phi$ that depends only on temperature, which comes from the potential energy of the atoms of a molecule, that is, $u=\nu a+\phi$. According to equation (47), the translational energy per molecule is $(3 / 2) k T$, which gives $a=(1 / 2) k T$, because there are three degrees of freedom related to translational motion. The heat capacities per molecule are $c_{v}=(\nu / 2) k+\phi^{\prime}$ and $c_{p}=c_{v}+k$, which is the relation analogous to 40 . The ratio $\gamma=c_{p} / c_{v}$ gives relation (54) where $e=2 \phi^{\prime} / k$. If the molecule is rigid, $e=0$ and

$$
\gamma=1+\frac{2}{\nu}
$$

From the value of $\gamma$ determined by Kundt and Warburg for mercury vapor, oliveira Boltzmann found from formula (54) that $\nu=3$ and $e=0$, confirming that a monatomic molecule acts like a material point, which has $\nu=3$ degrees of freedom 98. By analogy Boltzmann assumes that a diatomic molecule consists of two connected spheres, or a dumbbell, acting as two material points and thus having $\nu=5$ degrees of freedom [98]. When $\nu=5$, formula (55) gives $\gamma=7 / 5=1.4$, a value which is very close to the experimental values found for air and other diatomic gases, as shown in Table 3 and 4.

The rigid dumbbell model for a diatomic molecule was also proposed independently by Bosanquet in 1877 [99]. According to Bosanquet, such a molecule would have five equal parts of kinetic energy, three due to translation and two to rotation, given a ratio $K / U=3 / 5$, which from Clausius equation (49) Bosanquet oliveirafinds $\gamma=7 / 5=$ 1.4. This explanation, says Bosanquet, "is so obvious that it is impossible to suppose that it has not occurred before to the eminent men who have dealt with the subject" 99].

The theory of Boltzmann for diatomic molecules justifies law (1) of Delarive and Marcet by recognizing that "simple gases, and compound gases which result from the combination of simple gases without a change of volume" are diatomic gases. The volume specific heat at a constant pressure of a diatomic gas is $\bar{c}_{p}=C_{p} / V=(5 / 2)(N / V) k$. Since, from Avogadro law, $N / V=p / k T$ has the same values for all gases at the same temperature and pressure, then $\bar{c}_{p}$ has the same value for all diatomic gases, which is law (1) of Delarive and Marcet.

The ratio of specific heats was used by Rayleigh and Ramsay to establish that the gas argon was monatomic. After the discovery of the gas argon by them in 1894, they had reasons to believe that it was a chemical element. From the measurement of its density it was found that the molecular weight of argon was 39.8, if that of the oxygen is taken as 32 . The next problem was to determine whether the gas argon was diatomic like the other simple gases. To this end Ramsay, using the method of Kundt and Warburg, determined the ratio $\gamma$ of the specific heats from the measurement of the speed of sound. The value found was $\gamma=1.649$, which was distinct from $7 / 5$ of diatomic molecules, but close to $5 / 3$ of monatomic molecules, establishing argon as a monatomic gas with an atomic weight 39.8 [79].

\subsection{Equipartition theorem}

We have seen that the attempt by Maxwell to demonstrate the equipartition of energy from pure mechanics was not successful. The demonstrations that came later, by Maxwell [89], by Boltzmann 94], and by Watson 96] were based on the use of a probability distribution. Since the derivation of a probability distribution from pure mechanics has not been accomplished, one cannot either say that these derivations of the equipartition come from pure mechanics. In fact, the difficulty of deriving the probability distribution lead to the postulation of the distribution as occurred with the Gibbs microcanonical and canonical distribution of statistical mechanics of systems in thermodynamic equilibrium. Sometimes the equipartition of energy is referred to as a theorem. It is indeed a theorem of statistical mechanics but not a theorem of pure mechanics.

Next, we show that the equipartition can be derived from any probability distribution $P$ which is a function of the energy, or more precisely, of the Hamiltonian $\mathcal{H}$, which are the cases of the Gibbs microcanonical an canonical distribution. We denote by $q$ the collection of the coordinates and by $p$ the collection of their conjugate momenta and write

$$
P(q, p)=F(\mathcal{H}(q, p))
$$

that is, $P(q, p)$ depends on the dynamic variables $(q, p)$ through $\mathcal{H}(q, p)$. We consider an ideal gas so that $\mathcal{H}$ is a sum of terms $\mathcal{H}_{i}$, one for each molecule,

$$
\mathcal{H}=\sum_{i} \mathcal{H}_{i}
$$

We assume the following form for the molecule Hamiltonian

$$
\mathcal{H}_{i}=\sum_{j} A_{i j} p_{i j}^{2}+B_{i}
$$

where $A_{i j}$ and $B_{i}$ depend only on the coordinates corresponding to molecule $i$. Next we define new momenta $\xi_{i j}$ by

$$
\xi_{i j}=p_{i j} \sqrt{A_{i j}}
$$

The new coordinates $\eta_{i j}$ are chosen as the canonical conjugates of $\xi_{i j}$, which implies that the Jacobian of the transformation from the from the old to new variables equals the unity. In the new variables the Hamiltonian of a molecule is

$$
\mathcal{H}_{i}=\sum_{j} \xi_{i j}^{2}+C_{i}
$$


where $C_{i}$ depends only on the new coordinates $\eta_{i j}$, and the total Hamiltonian is

$$
\mathcal{H}=\sum_{i j} \xi_{i j}^{2}+C
$$

where $C$ is the sum of $C_{i}$. In the new variables, collectively denoted by $(\xi, \eta)$, the probability distribution is

$$
P(\xi, \eta)=F(\mathcal{H}(\xi, \eta)) .
$$

Now we integrate in $\eta$ to find the probability distribution of $\xi$,

$$
P^{*}(\xi)=F^{*}\left(\sum_{i j} \xi_{i j}^{2}\right),
$$

where $F^{*}$ is another function. That is, the probability distribution depends on $\xi$ only through the sum of $\xi_{i j}^{2}$. We see that $P^{*}(\xi)$ is invariant under the exchange of any pair of momenta $\xi_{i j}$, a property which allows us to conclude that the average $\left\langle\xi_{i j}^{2}\right\rangle$ is independent of $(i, j)$. Therefore the average $\left\langle A_{i j} p_{i j}^{2}\right\rangle$ is independent of $(i, j)$, which constitutes the equipartition of energy.

If the probability distribution is the Gibbs, canonical distribution, that is, if $F(\mathcal{H})$ is proportional to $e^{-\beta \mathcal{H}}$ where $\beta=1 / k T$ it follows that $\left\langle\xi_{i j}^{2}\right\rangle=k T / 2$, or

$$
\left\langle A_{i j} p_{i j}^{2}\right\rangle=\frac{1}{2} k T \text {. }
$$

The same reasoning can extended to the case where the molecules interact with each other, giving again the same equipartition of the kinetic energy (64).

Let us consider the case of a gas of diatomic molecules, each one being a rigid body of mass $m_{i}$ consisting of two material points, and $\nu=5$ degrees of freedom. The moment of inertia about the axis connecting the two material points is zero and the other two are equal and denoted by $I_{i}$. The Hamiltonian of each molecule is a sum of the translational and rotational, given by

$$
\mathcal{H}_{i}=\frac{1}{2 m_{i}}\left(p_{i 1}^{2}+p_{i 2}^{2}+p_{i 3}^{2}\right)+\frac{1}{2 I_{i}}\left(p_{i 4}^{2}+\frac{1}{\sin ^{2} \theta_{i}} p_{i 5}^{2}\right),
$$

where $p_{i 1}, p_{i 2}$, and $p_{i 3}$ are the Cartesian components of the linear momenta and $p_{i 4}$ and $p_{i 5}$ are the angular momenta.

From the equipartition theorem, it follows at once that

$$
\left\langle\frac{p_{i 1}^{2}}{2 m_{i}}\right\rangle=\left\langle\frac{p_{i 2}^{2}}{2 m_{i}}\right\rangle=\left\langle\frac{p_{i 3}^{2}}{2 m_{i}}\right\rangle=\left\langle\frac{p_{i 4}^{2}}{2 I_{i}}\right\rangle=\left\langle\frac{p_{i 5}^{2}}{2 I_{i} \sin ^{2} \theta_{i}}\right\rangle
$$

has the same value $k T / 2$ for any $i$. From this result it follows that the average energy per molecule will be $u=5 k T / 2$.

The reasoning above can be extended to the case where each molecule is a rigid body such as an spheroid, which has three rotational degrees of freedom in addition to the three translational degrees of freedom making a total of $\nu=6$ degrees of freedom. The resulting average energy per molecule is $u=6 k T / 2$.

\section{Maxwell and Boltzmann}

The model of a molecule used by Boltzmann 98 to describe a diatomic ideal gas was a rigid body with cylindrical symmetry, like an spheroid or a dumbbell. Such a rigid body has three rotational degrees of freedom. However, Boltzmann argued that the rotation about the axis of symmetry would not be activated by collisions so that the body would act like having two rotational degrees of freedom, as happens to the rigid molecular model analyzed in the previous section, consisting of two material points. The total degrees of freedom being $\nu=5$ gives for the ratio of specific heats the value $\gamma=7 / 5=1.4$, in agreement with the experimental results for diatomic gases. Boltzmann also explained that the vibrations need not to be taken into account because they occur only briefly during the collisions of two molecules, analogous to the vibration occurring when two billiard balls strike each other, thus making the molecule as if it were a rigid body 98 .

Although the explanation given by Boltzmann was in agreement with the experimental results, it was not accepted by Maxwell [100]. He argued that any extended rigid body of any shape has three rotational degrees of freedom in addition to the three translational degrees of freedom, making $\nu=6$, and $\gamma=4 / 3$. Moreover, if a molecule could vibrate, as the spectroscopy at that time showed, the value of $\gamma$, as given by formula (54), would be even smaller than $4 / 3$, incompatible with the experimental value for diatomic gases.

The reasoning of Maxwell, although correct from the point of view of Newtonian mechanics, did not explain the experimental results. The reasonings of Boltzmann explained the experimental results, but his explanation of the absence of both the rotation about the axis symmetry and vibration did not convince Maxwell. It is meaningful that the disagreement between these two physicists extended to their styles of communication, although they had a great appreciation for each other's work. Boltzmann said that Maxwell was difficult to understand because of its great brevity [94. Maxwell in his turn said that Boltzmann was hard to understand because of the opposite reason. Boltzmann's length was an equally stumbling-block to Maxwell 101.

The paradox of the specific heats was solved after the emergence of quantum mechanics, which treats the energy of rotation as well as that of vibration as quantized. Consider the rotation about the axis of symmetry of a spheroid or a dumbbell. According to quantum mechanics, the energy of rotation is quantized and given by

$$
E_{\ell}=\frac{1}{2 I} \hbar^{2} \ell(\ell+1),
$$

where $\ell=0,1,2 \ldots$ and $I$ is the moment of inertia around the axis of symmetry, and $\hbar$ is the Planck constant. If $I$ is small, the energy of the first excited state, $E_{1}=\hbar^{2} / I$, will be great. If the energy of collisions is not large enough, a molecule will not be raised to the excited states and 
remain in the ground state, acting as if it did not rotate about the axis of symmetry, as wished Boltzmann. This reasoning applies only to the rotation about the axis of symmetry. The rotation about the other two axes will occur because the other momenta of inertia are much greater than $I$.

The same quantum explanation can be extended to vibrational degrees of freedom. Consider one of the several modes of oscillation of a molecule characterized by a angular frequency $\omega$. The corresponding quantized energy of this mode is

$$
E_{n}=\hbar \omega\left(n+\frac{1}{2}\right)
$$

where $n=0,1,2, \ldots$. Again, if the energy of collisions is not large enough, a molecule will not be excited and will remain in the ground state, as if it did not vibrate, as wished Boltzmann.

\section{Conclusion}

We have given an account and a critical analysis of the development of laws concerning the ideal gas, comprising the period where the caloric theory was the prevailing theory of heat and the first decades after the emergence of thermodynamics. During this period, the elastic properties of gases and the specific heats, including their ratio, were the main concerns of the experimental investigation on gases. We have described the relation of the laws related to the elastic and thermal properties of gases among themselves and with the law of equipartition of energy.

The kinetic theory, as presented by Clausius and Maxwell in their respective first papers on the subject aimed to explain the macroscopic laws of gases by assuming that the molecules follows the laws of Newtonian mechanics. Indeed, it was possible to derive from these assumptions the law that relates pressure to the translational kinetic energy, given by equation (45). The law of equipartition of energy, related to translational motion, appeared in the papers of Waterston and Clausius on the kinetic theory. In a more general form, which included the rotational motion, the equipartition was formulated by Maxwell in connection with the kinetic theory. These three physicists showed, by the use of equation 45, that the Avogadro law is a consequence of translational equipartition of kinetic energy. From this result, it follows the ideal gas equation in the form $(43)$ or 42 .

The attempts to derive the equipartition of energy from pure mechanics, for instance, the translational equipartition given by equation (47), were not successful. However, the equipartition could be derived from statistical mechanics, which uses statistical description of a system. This approach was used by Maxwell and Boltzmann to derive the law of equipartition. Instead of presenting their derivation, we have carried out a simplified derivation starting from a probability distribution that depends only on the energy, which are the case of the Gibbs microcanonical and canonical distributions. This derivation reveals that the equipartition comes from the invariance of probability distribution under the exchange of two parts of the Hamiltonian. This is possible because the probability distribution depends on the dynamical variables through the Hamiltonian, a feature that describes systems in thermodynamic equilibrium.

It should be noted finally that the probabilistic distribution of statistical mechanics are introduced as fundamental principles, which means that they are not or need not to be derived from pure mechanics.

\section{Acknowledgement}

I wish to acknowledge helpful conversation with Vera Henriques about Mayer and his method to determine the mechanical equivalent of heat. The critical reading of the manuscript by Tânia Tomé is also acknowledged.

\section{References}

[1] B. Mahon, The Man that Changed Everything, The Life of James Clerk Maxwell (Wiley, Chichester, 2004).

[2] C. Cercignani, Ludwig Boltzmann, The Man Who Trusted Atoms (Oxford University Press, Oxford, 1998).

[3] J.H. Jeans, The Dynamical Theory of Gases (Cambridge Universiyt Press, Cambridge, 1904).

[4] D. Mckie and N.H.V. Heathcote, The Discovery of Specific and Latent Heats (Arnold, London, 1935).

[5] R. Fox, The Caloric Theory of Gases (Clarendon Press, Oxford, 1971)

[6] M.J. de Oliveira, Braz. J. Phys. 48, 299 (2018).

[7] M. Boas, Robert Boyle and Seventeenth-Century chemistry (Cambridge University Press, Cambridge, 1958).

[8] M. Crosland, Gay-Lussac Scientist and Bourgeois (Cambridge University Press, Cambridge, 1978).

[9] M. Morselli, Amedeo Avogadro, A Scientific Biography (Reidel, Dordrecht, 1984).

[10] B.S. Finn, Isis 55, 7 (1964).

[11] J.L. Hargrove, Journal of Nutrition 136, 2957 (2006).

[12] R. Boyle, A Defence of the Doctrine touching the Spring and Weight of the Air (Thomas Robinson, London, 1662).

[13] E. Mariotte, Discours de la Nataure de l'Air (Estienne Michallet, Paris, 1679).

[14] J.L. Gay-Lussac, Annales de Chimie 43, 137 (1802).

[15] J.B. Biot, Traité de Physique Expérimentale et Mathématique (Deterville, Paris, 1816), v. 1.

[16] S.D. Poisson, Connaissance des Tems ou des Mouvements Célestes à l'Usage des Astronomes et des Navigateurs pour l'an 1826, p. 257 (1823), available in https://gallica.bnf.fr/ark: /12148/bpt6k65109513/f5.image

[17] S. Carnot, Réflexions sur la Puissance Motrice du Feu et sur les Machines propes à Developper cette Puissance (Bachelier, Paris, 1824).

[18] E. Clapeyron, Journal de l'École Royale Polytechnique 14, 153 (1834).

[19] W.B. Jensen, J. Chem. Educ. 80, 731 (2003). 
[20] F. Rudberg, Annalen der Physik und Chemie 41, 271 (1837).

[21] R. Clausius, Annalen der Physik und Chemie 155, 368 (1850).

[22] V. Regnault, Annales de Chimie et de Physique 4, 5; 5, $52(1842)$.

[23] R. Clausius, Annalen der Physik und Chemie 116, 73 (1862).

[24] P.S. Laplace, Connaissance des Tems ou des Mouvements Célestes à l'Usage des Astronomes et des Navigateurs pour l'an 1825, p. 219 (1822), available in https:// gallica.bnf.fr/ark:/12148/bpt6k65057931

[25] J.B. Biot and F. Arago, Mémoires de la Classe des Sciences Mathématiques et Physiques de l'Institut National de France 7, 301 (1806).

[26] J.L. Gay-Lussac, Mémoires de physique et de chimie de la Societé d'Arcueil 2, 235 (1809).

[27] V. Regnault, Relation des Expériences pour Déterminer les Principales Lois et les Données Numèrique qui Entrent dans le Calcul des Machines à Vapeur (Firmin Didot, Paris, 1847).

[28] V. Regnault, Comptes Rendus Hebdomadaires des Séances de l'Académie des Sciences 36, 676 (1853).

[29] W.J.M. Rankine, Transactions of the Royal Society of Edinburgh 20, 147 (1853).

[30] R. Clausius, Annalen der Physik und Chemie 93, 481 (1854).

[31] R.J. Haüi, Traité Élémentaire de Physique (Courcier, Paris, 1806).

[32] F. Delaroche and J.E. Bérard, Annales de Chimie 85, 72 (1813).

[33] W.T. Haycraft, Transactions of the Royal Society of Edinburgh 10, 195 (1826).

[34] A. Delarive and F. Marcet, Annales de Chimie et de Physique 35, 5 (1827).

[35] A. Delarive and F. Marcet, Annales de Chimie et de Physique 41, 78 (1829).

[36] P.L. Dulong, Annales de Chimie et de Physique 41, 113 (1829).

[37] A. Delarive and F. Marcet, Annales de Chimie et de Physique 75, 113 (1840).

[38] V. Regnault, Relation des Expériences pour Déterminer les Lois et les Données Physiques Nécessaires au Calcul des Machines à Feu (Firmin Didot, Paris, 1862), v. 2.

[39] A.T. Petit and P.L. Dulong, Annales de Chimie et de Physique 10, 395 (1819).

[40] P.S. Laplace, Annales de Chimie et de Physique 3, 238 (1816).

[41] J.L. Lagrange, Méchanique Analitique (Desaint, Paris, 1788).

[42] J.B. Biot, Journal de Physique, de Chimie, d'Histoire Naturelle et des Arts 55, 173 (1802).

[43] S.D. Poisson, Journal de L'École Polytechnique 7, 319 (1808).

[44] S.D. Poisson, Annales de Chimie et de Physique 23, 337 (1823).

[45] C.B. Desormes and N. Clément, Journal de Physique 89, 321 (1819).

[46] E. Péclet, Traité Élémentaire de Physique (Hachette, Paris, 1847), v.1, 4ème éd.

[47] A. Masson, Annales de Chimie et de Physique 53, 257 (1858).
[48] J. Weisbach, Civilingenieur 5, 46 (1859).

[49] A. Cazin, Annales de Chimie et de Physique 66, 206 (1862).

[50] G. Simons, Philosophical Transactions 120, 209 (1830).

[51] G. Moll and A. Van Beek, Philosophical Transactions 114, 424 (1824).

[52] A. Bravais and C.F. Martins, Comptes Rendus des Séances de l'Académie des Sciences 20, 1164 (1844).

[53] J.R. Mayer, Annalen der Chemie und Pharmacie 42, 233 (1842).

[54] J.P. Joule, Philosophical Magazine 23, 263 (1843).

[55] J.P. Joule, Philosophical Transactions of the Royal Society 140, 61 (1850).

[56] J.P. Joule, Philosophical Magazine 26, 369 (1845).

[57] J.R. Mayer, Die organische Bewegung in ihrem Zusammenhange mit dem Stoffwechsel, Ein Beitrag zur Naturkunde (Drechsler, Heilbronn, 1845).

[58] J.P. Joule, Philosophical Magazine 31, 114 (1847).

[59] W.J.M. Rankine, Philosophical Magazine 5, 483 (1853).

[60] J. Le Conte, Philosophical Magazine 27, 1 (1864).

[61] J.L. Gay-Lussac, Mémoires de physique et de chimie de la Societé d'Arcueil 2, 207 (1809).

[62] J.J. Berzelius, Annals of Philosophy 2, 443; 3, 51 (1814).

[63] J. Dalton, A New System of Chemical Philosophy, Part I e II (Bickerstaff, London, 1808).

[64] S. Cannizzaro, Nuovo Cimento 7, 321 (1858).

[65] A. Naumann, Berichte der deutschen chemischen Gesellschaft zu Berlin 2, 690 (1869).

[66] A. Avogadro, Journal de Physique, de Chimie, d'Histoire naturelle et des Arts 73, 58 (1811).

[67] A.M. Ampère, Annales de Chimie 90, 43 (1814).

[68] J.B. Dumas, Annales de Chimie et de Physique 33, 337 (1826).

[69] M.A. Gaudin, Annales de Chimie et de Physique 52, 113 (1833).

[70] I. Guareschi, in Opere Scelte di Amedeo Avogadro, (Reale Accademia delle Scienze di Torino, Torino, 1911).

[71] T.M. Cole Jr., Isis 66, 334 (1975).

[72] L. Meyer, Die modernen Theorien der Chemie und ihre Bedeutung für die chemische Statik (Maruschke \& Berendt, Breslau, 1864).

[73] L. Meyer, Die modernen Theorien der Chemie und ihre Bedeutung für die chemische Statik, (Maruschke $\&$ Berendt, Breslau, 1872).

[74] W. Nernst, Theoretische Chemie vom Standpunkte der Avogadro'schen Regel und der Thermodynamik (Ferdinand Enke, Stuttgart, 1893).

[75] M. Planck, Verhandlungen der Deutschen Physikalischen Gesellschaft 2, 237 (1900).

[76] C.M. Guldberg, Comptes Rendus Hebdomadaires des Séances de l'Académie des Sciences 65, 941 (1867); 66, 39 (1868).

[77] A. Horstmann, Annalen der Chemie und Pharmacie 170, 192 (1873).

[78] W. Ostwald, Hand- und Hilfsbuch zur Ausführung physiko-chemischer Messungen (Wilhelm Engelmann, Leipzig, 1893).

[79] M.W. Travers, The Discovery of the Rare Gases (Arnold, London, 1928).

[80] R. Clausius, Annalen der Physik und Chemie 100, 353 (1857).

[81] A. Krönig, Annalen der Physik 99, 315 (1856). 
[82] J.J. Waterston, Philosophical Transactions 183, 1 (1893).

[83] J.J. Waterston, Report of the British Association for the Advancement of Science 21st meeting 1851, p. 6 (1852). available in https://www.biodiversitylibrary. org/page/13846822\#page/1/mode/1up p. 604.

[84] J.W. Strutt, Philosophical Transactions 183, 3 (1893).

[85] J.C. Maxwell, Philosophical Magazine 19, 19; 20, 21 (1860).

[86] E. Garber, S.G. Brush and C.W.F. Everitt Maxwell on Molecules and Gases (MIT Press, Cambridge, 1986).

[87] S.G. Brush, The Kind of Motion We Call Heat (NorthHolland, Amsterdam. 1986), v. 1.

[88] A. Walstad, American Journal of Physics 81, 555 (2013).

[89] J.C. Maxwell, Philosophical Transactions of the Royal Society of london 157, 49 (1867).

[90] J.C. Maxwell, Nature 8, 437 (1873).

[91] J.C. Maxwell, Nature 11, 357, 374 (1875).

[92] S.G. Brush, The Kind of Motion We Call Heat (NorthHolland, Amsterdam, 1986), v. 2.

[93] J.C. Maxwell, Transactions of the Cambridge Philosophical Society 12, 547 (1879).

[94] L. Boltzmann, Sitzungsberichte der kaiserlichen Akademie der Wissenschaften 58, 517 (1868).

[95] L. Boltzmann, Sitzungsberichte der kaiserlichen Akademie der Wissenschaften 63, 397 (1871).

[96] H.W. Watson, A Treatise on the Kinetic Theory of Gases (Clarendon Press, Oxford, 1876).

[97] A. Kundt and E. Warburg, Berichte der deutschen chemischen Gesellschaft 8, 945 (1875).

[98] L. Boltzmann, Sitzungsberichte der Kaiserlichen Akademie der Wissenschaften 74, 553 (1877).

[99] R.H.M. Bosanquet, Philosophical Magazine 3, 271; 4, 25 (1877).

[100] J.C. Maxwell, Nature 16, 242 (1877).

[101] C. G. Knott, in: Life and Scientific Work of Peter Guthrie Tait (Cambridge University Press, Cambridge, 1911), p. 114. 